\title{
ASYMPTOTIC MODELS FOR SCATTERING FROM UNBOUNDED MEDIA WITH HIGH CONDUCTIVITY
}

\author{
Houssem HADDAR $^{1}$ AND ARMin LECHLEITER ${ }^{1}$
}

\begin{abstract}
We analyze the accuracy and well-posedness of generalized impedance boundary value problems in the framework of scattering problems from unbounded highly absorbing media. We restrict ourselves in this first work to the scalar problem (E-mode for electromagnetic scattering problems). Compared to earlier works, the unboundedness of the rough absorbing layer introduces severe difficulties in the analysis for the generalized impedance boundary conditions, since classical compactness arguments are no longer possible. Our new analysis is based on the use of Rellich-type estimates and boundedness of $L 2$ solution operators. We also discuss some numerical experiments concerning these boundary conditions.
\end{abstract}

Mathematics Subject Classification. 35C20, 78A40.

Received February 22, 2009. Revised December 5, 2009.

Published online April 15, 2010.

\section{INTRODUCTION}

Time harmonic wave scattering from rough layers is an important problem in science and engineering, as it describes for instance scattering of electromagnetic waves from the ground when one models the earth as a rough stratified medium. In such a model, the moisture of soil causes absorption of the electromagnetic wave inside the ground, and thus naturally leads to a scattering problem for a rough absorbing layer. Since waves inside the absorbing part of the medium decay exponentially with respect to the distance to the layer's boundary, a lot of research has been carried out how to replace the wave scattering problem inside the absorbing layer by some easily handable absorbing boundary condition on the interface in between the absorbing layer and free space $[1,8,9,14,15]$. The aim of such a boundary condition is to set up an approximate scattering problem merely in the complement of the absorbing object, while still guaranteeing a reliable error bound on the solution of the approximate problem. This error bound depends on what we call the order of the boundary condition as well as on the magnitude of the absorption inside the layer. Indeed, we treat the magnitude of absorption as a parameter and expand the wave field in a power series with respect to the inverse of this parameter. Approximate boundary conditions are built after truncation of this series, the order of the conditions so obtained then corresponds to the truncation index. Truncation at order 0 simply leads to a Dirichlet boundary condition, which is naturally the formal limit condition as the absorption tends to infinity; truncation at order 1 leads to a (usual) impedance

\footnotetext{
Keywords and phrases. Scattering problems, unbounded domains, asymptotic models, generalized impedance boundary conditions, high conductivity.

${ }^{1}$ INRIA Saclay Île-de-France/École Polytechnique, Route de Saclay, 91128 Palaiseau Cedex, France.

haddar@cmap.polytechnique.fr; alechle@cmap.polytechnique.fr
} 
boundary condition. This is the reason why we call the condition arising from truncation at higher order generalized impedance boundary condition (GIBC).

In this paper, we analyse GIBCs for rough absorbing layers up to order 3 and shall restrict ourselves to the scalar problem (which corresponds in 2-D to the E-polarization of electromagnetic waves). While the construction of such conditions is rather analogous to the case of a bounded absorbing inhomogeneity, the error analysis is more complicated because classical compactness arguments fail due to the unboundedness of the domain. The difficulties are already obvious when one considers merely existence and uniqueness of solution for the approximate problems. For instance, for the case of a bounded obstacle, existence of solution for the time harmonic exterior Dirichlet or impedance scattering problem is known for a long time [13]. For the rough surface scattering problem with a Dirichlet boundary condition, corresponding results have only been achieved during the last decade, firstly by using integral equation approach $[4,5,16]$, and more recently, by using a variational approach in $[2,6]$. For scattering from rough infinite layers we also refer to recent results in [11]. For the variational theory on rough surface scattering, Rellich identities have been shown to be particularly useful since they provide a priori bounds on a solution to the scattering problem, thereby establishing existence and uniqueness of solution via an inf-sup condition.

When considering the Helmholtz equation involving a bounded scattering object, Rellich identities are a well-known tool to obtain explicit a priori bounds on solutions, see, e.g., [7]. However, existence theory for wave scattering problems for bounded objects does not necessarily require this technical tool since it can be based on Fredholm theory. However, analysis of scattering from unbounded objects, where Rellich's lemma on compact embeddings fails, cannot (at least not in an "easy" way) be based on Fredholm theory. In consequence, tools like Rellich identities that yield a priori estimates become crucial for existence theory.

Thus, it is not surprising that a priori bounds are also important for proving existence of solution to rough layer scattering problems involving higher order impedance boundary conditions. Moreover, they permit to construct a so-called bounded $L^{2}$ solution operator. This operator maps Dirichlet boundary data on the rough surface to the radiating solution of the Helmholtz equation taking this boundary data. We show that this operator has a bounded extension from square integrable functions on the interface into the space of square integrable functions in a layer of finite height above the interface.

The important role of this $L^{2}$ solution operator in our analysis is to replace compactness arguments present in earlier rigorous error analysis of generalized impedance boundary conditions. Since compact embeddings of Sobolev spaces do not hold in our unbounded setting, we cannot use such types of compactness arguments which are always present in earlier works on generalized impedance boundary conditions for bounded objects [9]. The $L^{2}$ solution operator does part of this job. The other part is mainly done by a Rellich identity for radiating solutions of the Helmholtz equation over a rough layer. Through our Rellich identity we are able to prove existence and uniqueness of solution to the rough layer scattering problem subject to generalized impedance boundary conditions up to order 3. Further, we show optimal error bounds for solutions to the approximate scattering problems involving our generalized impedance boundary conditions compared to the solution of the original scattering problem in the absorbing layer.

The structure of this paper is as follows. The first section is dedicated to the presentation of the mathematical setting of the scattering problem from unbounded rough surfaces and the introduction of some notation. Section 2 serves as a brief review of the main steps in deriving generalized impedance boundary conditions and required extensions to the case of rough surfaces. Afterwards, we provide abstract existence theory for generalized impedance boundary value problems on rough surfaces in Section 3, which has an interest in its own. Section 4 contains asymptotic analysis of Neumann-to-Dirichlet GBICs up to order 2; for the corresponding convergence result see Theorem 4.1. A more complicated condition of order 3 is analyzed in Section 5 , leading to an order-optimal convergence result in Theorem 5.4. In this section, we indicate in particular how a "stabilized" condition can be treated in a similar way as standard impedance boundary conditions through our abstract existence theory. 


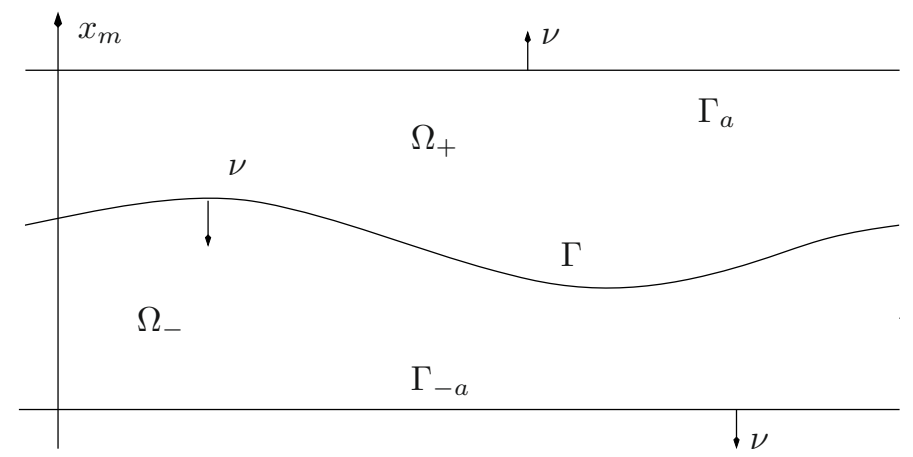

Figure 1. The geometry of the rough layer/rough surface problems. The domain $\Omega=\Omega_{+} \cup$ $\Gamma \cup \Omega_{-}$lies in between the two planes $\Gamma_{a}=\left\{x_{m}=a\right\}$ and $\Gamma_{-a}=\left\{x_{m}=-a\right\}$. In $\Omega$, the refractive index $n^{2}$ varies; while $n^{2}$ is Lipschitz continuous in $\Omega_{ \pm}$, the index may jump across $\Gamma$. The unit normal $\nu$ points out of $\Omega$ and on $\Gamma$ we choose $\nu$ to point downwards. The domain $\Omega_{R}=\{x \in \Omega,|\tilde{x}|<R\}$ is obtained from $\Omega$ by cut off in the lateral variables $\tilde{x}$.

\section{Problem Setting And notation}

Let us start with a brief description of the geometrical setting and our notation, such that we can afterwards present the problem mathematical setting in detail. Points in the Euclidean space $\mathbb{R}^{m}(m=2,3)$ are denoted by $x=\left(x_{1}, x_{2}, \ldots, x_{m}\right)^{\top}$ and sometimes it is convenient to write $x=\left(\tilde{x}, x_{m}\right)^{\top}$, that is, $\tilde{x}$ are the first $m-1$ coordinates of $x \in \mathbb{R}^{m}$. By $\mathbb{R}_{ \pm}^{m}:=\left\{x \in \mathbb{R}^{m}, x_{m} \gtrless 0\right\}$ we denote the upper and lower half space of $\mathbb{R}^{m}$ and the plane in between $\mathbb{R}_{ \pm}^{m}$ is $\Gamma_{0}=\left\{x \in \mathbb{R}^{m}, x_{m}=0\right\}$. More generally, $\Gamma_{a}=\left\{x=\left(x_{1}, x_{2}, \ldots, x_{m}\right)^{\top} \in \mathbb{R}^{m}, x_{m}=a\right\}$ for $a \in \mathbb{R}$. The half space above and below $\Gamma_{a}$ is denoted by $U_{a}^{ \pm}:=\left\{x \in \mathbb{R}^{m}, x_{m} \gtrless a\right\}$. The domain $\Omega:=\left\{x \in \mathbb{R}^{m},-a<x_{m}<a\right\}$ is partitioned into two parts $\Omega_{ \pm}:=\left\{x \in \Omega, x_{n} \gtrless f(\tilde{x})\right\}$ by the interface $\Gamma:=\left\{x \in \mathbb{R}^{m}, f(\tilde{x})=x_{m}\right\}$, given by a function $f: \mathbb{R}^{m-1} \rightarrow \mathbb{R},-a<f<a$. By $C^{\ell, 1}\left(\mathbb{R}^{m-1}, \mathbb{R}\right)$ we denote the Banach space of $\ell$-times continuously differentiable real-valued functions such that all $\ell$ th partial derivatives are Lipschitz continuous. A norm on this space is given by

$$
\|f\|=\sup _{|\alpha| \leq \ell}\left\|\frac{\partial^{|\alpha|} f}{\partial x^{\alpha}}\right\|_{L^{\infty}(\Omega)}+\sup _{|\alpha|=\ell, x \neq y} \frac{\left|\frac{\partial^{\ell} f}{\partial x^{\alpha}}(x)-\frac{\partial^{\ell} f}{\partial x^{\alpha}}(y)\right|}{|x-y|}, \quad \ell \in \mathbb{N}_{0} .
$$

If $f \in C^{\ell, 1}\left(\mathbb{R}^{m-1}, \mathbb{R}\right)$ we say that $\Gamma$ is of class $C_{\mathrm{b}}^{\ell, 1}$,

$$
\Gamma \in C_{\mathrm{b}}^{\ell, 1}, \quad \ell \in \mathbb{N}_{0} .
$$

We always suppose that $\Gamma \in C_{\mathrm{b}}^{0,1}$ is a Lipschitz surface; however, note that the asymptotic analysis of the GIBCs will require much more smoothness of $\Gamma$. For simplicity, we also introduce $\Omega_{R}:=\left\{x \in \Omega, x_{1}^{2}+\cdots+x_{m-1}^{2}<R^{2}\right\}$. The exterior unit normal field to $\Omega$ and $\Omega_{R}$ is called $\nu$; on $\Gamma$ we choose the unit normal field $\nu$ to point downwards into $\Omega_{-}$. The boundary of $\Omega_{R}$ is $C_{R}:=\partial \Omega_{R}$. We split $C_{R}=C_{R}^{+} \cup C_{R}^{-}$with $C_{R}^{-}:=\left\{x \in \Gamma_{-a}, x_{1}^{2}+\cdots+\right.$ $\left.x_{m-1}^{2}<R^{2}\right\}$ and define $M_{R}:=C_{R} \cap \Omega$. We refer to Figure 1 for a sketch of the geometry of the problem.

By $[u]_{\Gamma}$ we denote the jump of a function $u$ across the interface $\Gamma$, that is, $[u]_{\Gamma}=\left.u\right|_{\Gamma} ^{+}-\left.u\right|_{\Gamma} ^{-}$where $\left.u\right|_{\Gamma} ^{ \pm}$is the limit taken from $\Omega_{ \pm}$. The inner product of $L^{2}(\Gamma)$ is denoted as $\langle\cdot, \cdot\rangle_{L^{2}(\Gamma)}$. Let us also remark that the square root of the complex unit $-\mathrm{i}$ in the fourth quadrant is denoted by $\alpha=(1-\mathrm{i}) / \sqrt{2}$. All fields in this paper are time harmonic, that is, their time dependence is $\exp (-\mathrm{i} \omega t)$ for frequency $\omega>0$, and this dependence will always be suppressed. The wave number $k>0$ is defined as $k=\omega / c$ with $c>0$ the speed of sound in vacuum. 
Now we turn to the mathematical formulation of the rough layer problem. We describe the medium of propagation by a refractive index function $n^{2}$, which is assumed to be a real valued function in $\Omega_{+}$and of the special form

$$
n^{2}=1+\frac{\mathrm{i}}{k^{2} \varepsilon^{2}} \quad \text { in } \Omega_{-} \text {for } \varepsilon>0
$$

The (small) parameter $\varepsilon$ is proportional to $1 / \sqrt{k \sigma}$ where $\sigma$ denotes the conductivity of the lower medium and represents the so-called skin depth. This parameter therefore controls the width of the region that is penetrable by the incident wave. Concerning smoothness, we always require that $n^{2} \in C^{0,1}\left(\overline{\Omega_{+}}, \mathbb{R}\right)$ and for some statements we even require more smoothness of $\left.n^{2}\right|_{\Omega_{+}}$. Also, we always suppose that $n^{2} \geq c_{0}>0$ in $\Omega_{+}$. In the upper half space $U_{a}^{+}$we suppose $n^{2}$ to equal a positive constant $n_{+}^{2} \geq c_{0}$ such that $\left.n^{2}\right|_{\Gamma_{a}}=n_{+}^{2}$, while, obviously, $n^{2}$ equals $n_{-}^{2}:=1+\mathrm{i} /\left(k \varepsilon^{2}\right)$ in $U_{-a}^{-}$. Important for existence of solutions to rough layer scattering problems is the further assumption that $\partial n^{2} / \partial x_{m} \geq 0$ in $\Omega_{+}$. All these assumptions are supposed to hold throughout the paper. We remark that we could also deal with a refractive index whose real part varies in $\Omega_{-}$, but for simplicity do not consider this case here. In contrast, no complications arise from treating a varying index in $\Omega_{+}$, and since this case is of some importance for modelling coated conducting layers we decided to include it in the analysis.

The total field due to a local time harmonic source $g$ supported in $\Omega_{+}$satisfies the Helmholtz equation

$$
\Delta u+k^{2} n^{2} u=g \quad \text { in } \mathbb{R}^{m},
$$

subject to the additional assumption that $u$ and its normal derivative are continuous over the interface $\Gamma$ where the index of refraction $n^{2}$ jumps, and a radiation condition in $U_{ \pm a}^{ \pm}$. We note that the solution $u$ to the above problem will depend on $\varepsilon$ and denote $u=u^{\varepsilon}$.

Let us now introduce the radiation condition imposed on $u^{\varepsilon}$. As shown in [2], the Fourier transform

$$
\mathcal{F}: L^{2}\left(\mathbb{R}^{m-1}\right) \rightarrow L^{2}\left(\mathbb{R}^{m-1}\right), \quad \mathcal{F} \phi(\xi)=(2 \pi)^{-(m-1) / 2} \int_{\mathbb{R}^{m-1}} \mathrm{e}^{-\mathrm{i} \tilde{x} \cdot \xi} \phi(\tilde{x}) \mathrm{d} \tilde{x}, \quad \xi \in \mathbb{R}^{m-1}
$$

for $\phi \in L^{2}\left(\mathbb{R}^{m-1}\right) \cap L^{1}\left(\mathbb{R}^{m-1}\right)$, allows to explicitly compute a Dirichlet-to-Neumann operator, mapping Dirichlet values $\phi$ on $\Gamma_{a}$ to the Neumann boundary values of the unique radiating solution $u$ in $U_{a}^{+}$taking Dirichlet trace values $\phi$ on $\Gamma_{a}$. Construction of this operator relies on the following representation formula for $u^{\varepsilon}$,

$$
u^{\varepsilon}(x)=(2 \pi)^{-(m-1) / 2} \int_{\mathbb{R}^{m-1}} \exp \left(\mathrm{i}\left(\left(x_{m}-a\right) \sqrt{k^{2} n_{+}^{2}-\xi^{2}}+\tilde{x} \cdot \xi\right)\right) \mathcal{F}\left(\left.u\right|_{\Gamma_{a}}\right)(\xi) \mathrm{d} \xi
$$

for $x \in U_{a}^{+}$. Note that this representation is a superposition of upwards propagating plane waves. Computing the normal derivative of the latter expression reveals that the Dirichlet-to-Neumann operator $T_{n_{+}^{2}}^{+}: H^{1 / 2}\left(\Gamma_{a}\right) \rightarrow$ $H^{-1 / 2}\left(\Gamma_{a}\right)$ is given by

$$
\left(T_{n_{+}^{2}}^{+} \phi\right)(\tilde{x})=\mathrm{i}(2 \pi)^{-(m-1) / 2} \int_{\mathbb{R}^{m-1}} \sqrt{k^{2} n_{+}^{2}-\xi^{2}} \exp (\mathrm{i} \tilde{x} \cdot \xi) \mathcal{F} \phi(\xi) \mathrm{d} \xi
$$

and it is shown in [2] that $T_{n_{+}^{2}}^{+}$is bounded from $H^{1 / 2}\left(\Gamma_{a}\right)$ to $H^{-1 / 2}\left(\Gamma_{a}\right)$. A similar analysis shows that the corresponding Dirichlet-to-Neumann operator $T_{n_{-}^{2}}^{-}$on $\Gamma_{-a}$ is given by the very same expression (replacing of course $n_{+}^{2}$ by $n_{-}^{2}$ ). This is due to the fact that the expansion of $u^{\varepsilon}$ in $U_{-a}^{-}$consists of downwards propagating 
evanescent waves. From the representation of $T_{n_{+}^{2}}^{+}$as a Fourier multiplier we note that

$$
\begin{aligned}
\operatorname{Im} \int_{\Gamma_{a}} \overline{u^{\varepsilon, 3}} T_{n_{+}^{2}}^{+}\left(u^{\varepsilon, 3}\right) \mathrm{d} s & =\operatorname{Im}\left(\mathrm{i} \int_{\mathbb{R}^{m-1}} \sqrt{k^{2}-\xi^{2}}\left|\mathcal{F}\left(\left.u^{\varepsilon, 3}\right|_{\Gamma_{a}}\right)\right|^{2} \mathrm{~d} s\right) \\
& =\int_{|\xi|<k} \sqrt{k^{2}-\xi^{2}}\left|\mathcal{F}\left(\left.u^{\varepsilon, 3}\right|_{\Gamma_{a}}\right)\right|^{2} \mathrm{~d} s \geq 0
\end{aligned}
$$

and a similar computation shows that $-\operatorname{Re} \int_{\Gamma_{a}} \overline{u^{\varepsilon, 3}} T_{n_{+}^{2}}^{+}\left(u^{\varepsilon, 3}\right) \mathrm{d} s \geq 0$; again, the same inequalities holds for $T_{n_{-}^{2}}^{-}$.

The two Dirichlet-to-Neumann operators allow to frame the wave scattering problem under investigation variationally in the domain $\Omega$. Due to the homogeneous jump conditions $[u]_{\Gamma}=[\partial u / \partial \nu]_{\Gamma}=0$ it makes sense to seek a solution $u^{\varepsilon} \in H^{1}(\Omega)$. Twice (formally) applying Green's identity in $\Omega_{ \pm}$shows that

$$
\int_{\Omega}\left(\nabla u^{\varepsilon} \cdot \nabla \bar{v}-k^{2} n^{2} u^{\varepsilon}\right) \bar{v} \mathrm{~d} x-\int_{\Gamma_{a}} \bar{v} T_{n_{+}^{2}}^{+}\left(u^{\varepsilon}\right) \mathrm{d} s-\int_{\Gamma_{-a}} \bar{v} T_{n_{-}^{2}}^{-}\left(u^{\varepsilon}\right) \mathrm{d} s=-\int_{\Omega} g \bar{v} \mathrm{~d} x
$$

for all $v \in H^{1}(\Omega)$. Existence of a unique solution $u^{\varepsilon} \in H^{1}(\Omega)$ to this problem has been shown in [11].

We now introduce the Neumann-to-Dirichlet operator $D_{\varepsilon}$ on $\Gamma$ which maps $\phi \in H^{-1 / 2}(\Gamma)$ to the Dirichlet boundary values $v^{\varepsilon}$ on $\Gamma$ of the unique solution to

$$
\Delta v^{\varepsilon}+\left(k^{2}+\frac{\mathrm{i}}{\varepsilon^{2}}\right) v^{\varepsilon}=g \quad \text { in } \Omega_{-}, \quad \frac{\partial v^{\varepsilon}}{\partial \nu}=T_{n_{-}^{2}}^{-}\left(v^{\varepsilon}\right) \quad \text { on } \Gamma_{-a}, \quad \frac{\partial v^{\varepsilon}}{\partial \nu}=-\phi \quad \text { on } \Gamma .
$$

Due to absorption, the map $D_{\varepsilon}: \phi \mapsto v^{\varepsilon} \in H^{1 / 2}(\Gamma)$ is well defined and bounded. Of course, the restriction $\left.u^{\varepsilon}\right|_{\Omega_{+}}$solves

$$
\Delta u^{\varepsilon}+k^{2} u^{\varepsilon}=g \quad \text { in } \Omega_{+}, \quad \frac{\partial u^{\varepsilon}}{\partial \nu}=T_{n_{+}^{2}}^{+}\left(u^{\varepsilon}\right) \quad \text { on } \Gamma_{a}, \quad u^{\varepsilon}+D_{\varepsilon}\left(\frac{\partial u^{\varepsilon}}{\partial \nu}\right)=0 \quad \text { on } \Gamma .
$$

Roughly speaking, the idea of a generalized impedance boundary condition is now to construct a (formal) expansion of $D_{\varepsilon}$ in terms of $\varepsilon$ and to obtain explicitly computable boundary operators replacing $D_{\varepsilon}$ in (1.5). These approximations to $D_{\varepsilon}$ will introduce a certain error which we will show to be bounded in terms of powers of $\varepsilon$, the actual power depending on the truncation of the asymptotic development of $D_{\varepsilon}$. To conclude this brief outlook, as an approximation to the restriction $\left.u^{\varepsilon}\right|_{\Omega_{+}}$we are going to study problems of the following form,

$$
\Delta u^{\varepsilon, p}+k^{2} n^{2} u^{\varepsilon, p}=g \quad \text { in } \Omega_{+}, \quad \frac{\partial u^{\varepsilon, p}}{\partial \nu}=T_{n_{+}^{2}}^{+}\left(u^{\varepsilon, p}\right) \quad \text { on } \Gamma,
$$

together with

$$
u^{\varepsilon, p}+D_{\varepsilon, p}\left(\frac{\partial u^{\varepsilon, p}}{\partial \nu}\right)=0 \quad \text { on } \Gamma
$$

for certain Neumann-to-Dirichlet operators $D_{\varepsilon, p}$ of order $p \in\{0,1,2,3\}$, which will be constructed and analyzed in detail.

\section{Formal COnstruction of GIBCs}

As a brief introduction to the construction of generalized impedance boundary conditions, we recall some basic ideas, definitions and computations from [9]. The formal computation of these conditions for rough layers is the same as for bounded absorbing inclusions and we can skip most of the computations. Assume that $\Gamma \in C_{\mathrm{b}}^{1,1}$ and let $\Omega_{-}^{\delta}:=\left\{x \in \Omega_{-}\right.$, $\left.\operatorname{dist}(x, \Gamma)<\delta\right\}$ for $\delta>0$ small enough such that each point $x \in \Omega_{-}^{\delta}$ has 
a unique representation $x=x_{\Gamma}+q \nu$, with $x_{\Gamma} \in \Gamma$ and $q>0$. Recall that the unit normal $\nu$ on $\Gamma$ was defined to point into $\Omega_{-}$. The parameter $\delta$ is fixed from now on and we also fix a cut off function $\chi \in C^{\infty}\left(\Omega_{-}\right)$such that $\chi=1$ in $\Omega_{-}^{\delta / 2}, \chi=0$ in $\Omega_{-}^{\delta}$ as well as $0 \leq \chi \leq 1,|\nabla \chi| \leq C$ and $|\Delta \chi| \leq C$ in $\Omega_{-}$.

The starting point for the construction of generalized impedance boundary conditions is the assumption that the exact solution $u^{\varepsilon}$ of the scattering problem (1.4) with parameter $\varepsilon>0$ can be written as

$$
u^{\varepsilon}(x)=u_{+}^{0}(x)+\varepsilon u_{+}^{1}(x)+\varepsilon^{2} u_{+}^{2}(x)+\ldots, \quad x \in \Omega_{+},
$$

for functions $u_{+}^{\ell}: \Omega_{+} \rightarrow \mathbb{C}$ and

$$
\chi u^{\varepsilon}(x)=u_{-}^{0}\left(x_{\Gamma}, q / \varepsilon\right)+\varepsilon u_{-}^{1}\left(x_{\Gamma}, q / \varepsilon\right)+\varepsilon^{2} u_{-}^{2}\left(x_{\Gamma}, q / \varepsilon\right)+\ldots, \quad x=x_{\Gamma}+q \nu \in \Omega_{-}^{\delta},
$$

with functions $u_{-}^{\ell}: \Gamma \times \mathbb{R}_{+} \rightarrow \mathbb{C}$ such that $\lim _{\eta \rightarrow \infty} u_{-}^{\ell}\left(x_{\Gamma}, \eta\right)=0$ for $x_{\Gamma} \in \Gamma$. From this expansion we will in the sequel construct boundary conditions which allow to truncate problem (1.4) at $\Gamma$, introducing an error that can be controlled in powers of $\varepsilon$. Following [9], we set

$$
\tilde{u}_{-}^{\varepsilon}\left(x_{\Gamma}, \eta\right)=u_{-}^{0}\left(x_{\Gamma}, \eta\right)+\varepsilon u_{-}^{1}\left(x_{\Gamma}, \eta\right)+\varepsilon^{2} u_{-}^{2}\left(x_{\Gamma}, \eta\right)+\ldots, \quad x_{\Gamma} \in \Gamma, \eta>0 .
$$

Since $u^{\varepsilon}$ solves the Helmholtz equation, all functions $u_{+}^{\ell}$ also need to satisfy this equation. Moreover, starting from the Helmholtz equation (1.1) one can compute a differential equation for $\tilde{u}_{-}^{\varepsilon}$ of the form

$$
\left(-\frac{\partial^{2}}{\partial \eta^{2}}-\mathrm{i}\right) \tilde{u}_{-}^{\varepsilon}=\sum_{\ell=1}^{8} \varepsilon^{\ell} A_{\ell} \tilde{u}_{-}^{\varepsilon} \quad \text { on } \Gamma \times \mathbb{R}_{+},
$$

where the $A_{\ell}$ are differential operators in $\left(x_{\Gamma}, \eta\right)$ independent of $\varepsilon$; see [9] for details. Substituting the latter equation into (2.2) shows that

$$
\left(-\frac{\partial^{2}}{\partial \eta^{2}}-\mathrm{i}\right) u_{-}^{p}=\sum_{\ell=1}^{8} A_{\ell} u_{-}^{p-\ell} \quad \text { in } \Gamma \times \mathbb{R}_{+}
$$

where $u_{-}^{p-\ell}=0$ for $p-\ell<0$. Let us think of this equation as a family of second order ordinary differential equations in $\eta$ with parameter $x_{\Gamma}$. Coupling of (2.3) with the expansion (2.1) of $u^{\varepsilon}$ in $\Omega_{+}$yields a boundary condition at $\eta=0$ and together with the decay condition for $u_{-}^{\ell}$ we can solve (2.3). In more detail, equating terms in (2.1) and (2.2) which share the same powers of $\varepsilon$ either offers the possibility of coupling the Dirichlet traces,

or coupling of the normal derivatives,

$$
u_{+}^{p}\left(x_{\Gamma}\right)=u_{-}^{p}\left(x_{\Gamma}, 0\right) \quad \text { for } x_{\Gamma} \in \Gamma,
$$

$$
\frac{\partial u_{+}^{p-1}}{\partial \nu}\left(x_{\Gamma}\right)=\frac{\partial u_{-}^{p}}{\partial \eta}\left(x_{\Gamma}, 0\right) \quad \text { for } x_{\Gamma} \in \Gamma .
$$

We concentrate in this paper on the second option, which leads to Neumann-to-Dirichlet impedance boundary conditions, and which is somewhat more natural due to the shift of the index. The first option (2.4) results in Dirichlet-to-Neumann impedance boundary conditions which will not be considered here; we refer to [9] where formulas for Dirichlet-to-Neumann impedance boundary conditions for bounded obstacles are provided. Those boundary conditions can be analyzed in the rough surface context by the tools provided in the present paper.

The differential equations in $\eta$ in (2.3) together with (2.5) and the decay condition $u_{-}^{p}\left(x_{\Gamma}, \eta\right) \rightarrow 0$ as $\eta \rightarrow \infty$ can be explicitly solved in the form

$$
u_{-}^{p}\left(x_{\Gamma}, \eta\right)=P_{x_{\Gamma}}^{p}(\eta) \mathrm{e}^{-\alpha \eta}, \quad \eta>0, p \in \mathbb{N}_{0},
$$


with a polynomial $P_{x_{\Gamma}}^{p}$ of degree $p$ depending on $\partial u_{-}^{\ell} / \partial \nu, \ell \in\{0,1, \ldots, p-1\}$. There holds a recurrence relation of order 8 for the $P_{x_{\Gamma}}^{p}$, which we will however not give here, but only give the explicit form of the $u_{-}^{p}$ for $p \in\{0,1,2,3\}$. Before citing the result of this computation in [9], equations (4.26)-(4.29), we remark that we denote Gauss and mean curvature on $\Gamma$ by $G$ and $H$, respectively; we also assume familiarity of the reader with standard surface differential operators such as the surface gradient $\nabla_{\Gamma}$ and the surface Laplacian $\Delta_{\Gamma}$, see, e.g., [13] for details. Using the abbreviation $\alpha=(1-\mathrm{i}) / \sqrt{2}$ there holds

$$
\begin{aligned}
u_{-}^{0}\left(x_{\Gamma}, \eta\right)= & , \\
u_{-}^{1}\left(x_{\Gamma}, \eta\right)= & -\frac{1}{\alpha} \frac{\partial u_{+}^{0}}{\partial \nu}\left(x_{\Gamma}\right) \mathrm{e}^{-\alpha \eta}, \\
u_{-}^{2}\left(x_{\Gamma}, \eta\right)= & \left(-\frac{1}{\alpha} \frac{\partial u_{+}^{1}}{\partial \nu}\left(x_{\Gamma}\right)+\frac{H}{\alpha^{2}} \frac{\partial u_{+}^{0}}{\partial \nu}\left(x_{\Gamma}\right)+\eta \frac{H}{\alpha} \frac{\partial u_{+}^{0}}{\partial \nu}\left(x_{\Gamma}\right)\right) \mathrm{e}^{-\alpha \eta}, \\
u_{-}^{3}\left(x_{\Gamma}, \eta\right)= & {\left[-\frac{1}{\alpha} \frac{\partial u_{+}^{2}}{\partial \nu}\left(x_{\Gamma}\right)+\frac{H}{\alpha^{2}} \frac{\partial u_{+}^{1}}{\partial \nu}\left(x_{\Gamma}\right)-\frac{1}{2 \alpha^{3}}\left(3 H^{2}-G+k^{2}\right) \frac{\partial u_{+}^{0}}{\partial \nu}\left(x_{\Gamma}\right)\right.} \\
& -\frac{1}{2 \alpha^{3}} \Delta_{\Gamma}\left(\frac{\partial u_{+}^{0}}{\partial \nu}\right)+\eta\left(\frac{H}{\alpha} \frac{\partial u_{+}^{1}}{\partial \nu}\left(x_{\Gamma}\right)-\frac{1}{2 \alpha^{2}}\left(\Delta_{\Gamma}+3 H^{2}-G+k^{2}\right) \frac{\partial u_{+}^{0}}{\partial \nu}\left(x_{\Gamma}\right)\right) \\
& \left.+\eta^{2} \frac{1}{2 \alpha}\left(G-3 H^{2}\right) \frac{\partial u_{+}^{0}}{\partial \nu}\left(x_{\Gamma}\right)\right] \mathrm{e}^{-\alpha \eta} .
\end{aligned}
$$

We are going to show in Lemma (4.2) that the functions $u_{+}^{p}$ are well defined in $H^{1}\left(\Omega_{+}\right)$under some smoothness assumptions on the data.

Now we can derive a first version of generalized impedance boundary conditions. For $p \in\{0,1,2\}$ these will be the ones for which we prove convergence of optimal order in Section 4. For $p=3$, sophisticated further manipulations of the impedance boundary condition are necessary to derive such convergence.

The basic idea behind an impedance boundary condition of order $p$ is truncation of the expansion (2.1) of $u^{\varepsilon}$ at $\ell=p$,

$$
\tilde{u}^{\varepsilon, p}=\sum_{\ell=0}^{p} \varepsilon^{\ell} u_{+}^{\ell} \quad \text { in } \Omega_{+} .
$$

In view of (4.2) we get that $\tilde{u}^{\varepsilon, p}=\sum_{\ell=0}^{p} \varepsilon^{\ell} u_{-}^{\ell}(\cdot, 0)$ on $\Gamma$. Since $u_{-}^{\ell}(\cdot, 0)$ is explicitly given in $(2.6)-(2.9)$ in terms of the normal derivatives $\partial u_{+}^{\ell} / \partial \nu$, we can plug in these formulas into the last equation to obtain for $\ell=0$ that $\tilde{u}^{\varepsilon, 0}=0$ and for $\ell=1$ that

$$
\tilde{u}^{\varepsilon, 1}+\varepsilon \frac{1}{\alpha} \frac{\partial u_{+}^{0}}{\partial \nu}\left(x_{\Gamma}\right)=0, \quad \text { that is, } \quad \tilde{u}^{\varepsilon, 1}+\varepsilon \frac{1}{\alpha} \frac{\partial \tilde{u}^{\varepsilon, 1}}{\partial \nu}\left(x_{\Gamma}\right)=\varepsilon^{2} \frac{1}{\alpha} \frac{\partial \tilde{u}^{\varepsilon, 1}}{\partial \nu} .
$$

Generally speaking, we find a tangential differential operator $D_{\varepsilon, p}$ acting on $\Gamma$ such that

$$
\tilde{u}^{\varepsilon, p}+D_{\varepsilon, p}\left(\frac{\partial \tilde{u}^{\varepsilon, p}}{\partial \nu}\right)=\varepsilon^{p+1} r^{\varepsilon, p}, \quad p \in\{0,1,2,3\}
$$

for functions $r^{\varepsilon, p}$ which are explicitly given in [9], equation (4.34) for $p \in\{0,1,2,3\}$ as

$$
\begin{aligned}
r^{\varepsilon, 0}=0, \quad r^{\varepsilon, 1} & =\frac{1}{\alpha} \frac{\partial u_{+}^{1}}{\partial \nu}, \quad r^{\varepsilon, 2}=\frac{1}{\alpha} \frac{\partial u_{+}^{2}}{\partial \nu}-\mathrm{i} H \frac{\partial}{\partial \nu}\left(u_{+}^{1}+\varepsilon u_{+}^{2}\right), \\
r^{\varepsilon, 3} & =\frac{1}{\alpha} \frac{\partial u_{+}^{3}}{\partial \nu}-\mathrm{i} H \frac{\partial}{\partial \nu}\left(u_{+}^{1}+\varepsilon u_{+}^{2}\right)-\frac{1}{2}\left[\Delta_{\Gamma}+3 H^{2}-G+k^{2}\right]\left(\frac{\partial}{\partial \nu}\left(u_{+}^{1}+\varepsilon u_{+}^{2}+\varepsilon^{2} u_{+}^{3}\right)\right) .
\end{aligned}
$$


The differential operators $D_{\varepsilon, p}$ are given by [9], equations (3.5)-(3.8)

$D_{\varepsilon, 0}=0, \quad D_{\varepsilon, 1}=\frac{\varepsilon}{\alpha}, \quad D_{\varepsilon, 2}=\frac{\varepsilon}{\alpha}-\mathrm{i} \varepsilon^{2} H, \quad D_{\varepsilon, 3}=\frac{\varepsilon}{\alpha}-\mathrm{i} \varepsilon^{2} H-\varepsilon^{3} \frac{\alpha}{2}\left(\Delta_{\Gamma}+3 H^{2}-G+k^{2}\right)$.

Neglecting the small right hand side in (2.11), we define an approximation $u^{\varepsilon, p}, p=0,1,2$, of $u^{\varepsilon}$, solution of (1.4), by

$$
\begin{aligned}
\Delta u^{\varepsilon, p}+k^{2} n^{2} u^{\varepsilon, p}=g \quad \text { in } \Omega_{+}, \quad \frac{\partial u^{\varepsilon, p}}{\partial \nu}=T_{n_{+}^{2}}^{+}\left(u^{\varepsilon, p}\right) \quad \text { on } \Gamma_{a} \\
u^{\varepsilon, p}+D_{\varepsilon, p}\left(\frac{\partial u^{\varepsilon, p}}{\partial \nu}\right)=0 \quad \text { on } \Gamma, \quad \text { for } p=0,1,2 .
\end{aligned}
$$

Note that $u^{\varepsilon, 3}$ will be defined via a different (refined) boundary value problem in Section 5 . The function $u^{\varepsilon, p}$ solves a boundary value problem merely posed in $\Omega_{+}$. Well-posedness of this problem, as well as the approximation of $\left.u^{\varepsilon}\right|_{\Omega_{+}}$by $u^{\varepsilon, p}$ is subject of the following sections. However, at least morally we already note from (2.11) that the condition of order $p$ will introduce an error $\left\|u^{\varepsilon, p}-u^{\varepsilon}\right\|$ which is $\mathcal{O}\left(\varepsilon^{p+1}\right)$.

\section{Generalized impedance boundary value problems for rough Structures}

We start with some results on existence and uniqueness for Dirichlet scattering problems in $\Omega_{+}$, which are then used to establish an $L^{2}$ bound for the solution operator of the Dirichlet problem for a rough surface. This bound is very helpful to develop a general existence theory for generalized impedance boundary value problems on rough surfaces, which is the main result of this section.

The following Rellich identity [11], Section 7, is valid for any solution $u$ in $H^{2}\left(\Omega_{+}\right)$of the Helmholtz equation $\Delta u+k^{2} n^{2} u=g$, subject to the boundary condition $u=0$ on $\Gamma$ and the radiation condition $\partial u / \partial \nu=T_{n_{+}^{2}}^{+}(u)$ on $\Gamma_{a}$,

$$
\begin{aligned}
\int_{\Omega_{+}}\left(2\left|\frac{\partial v}{\partial x_{m}}\right|^{2}+\right. & \left.k^{2}\left(x_{m}+a\right) \frac{\partial n^{2}}{\partial x_{m}}|v|^{2}\right) \mathrm{d} x+2 a \int_{\Gamma_{a}}\left(|\nabla v|^{2}-2\left|\frac{\partial v}{\partial \nu}\right|^{2}-k^{2} n^{2}|v|^{2}\right) \mathrm{d} s \\
& -\int_{\Gamma_{a}} \bar{v} T_{n_{+}^{2}}^{+}(v) \mathrm{d} s+\int_{\Gamma}\left(x_{m}+a\right)\left(\nu_{m}|\nabla v|^{2}-2 \operatorname{Re}\left(\frac{\partial v}{\partial x_{m}} \frac{\partial \bar{v}}{\partial \nu}\right)-\nu_{m} k^{2}|v|^{2}\right) \mathrm{d} s \\
& =-2 \int_{\Gamma}\left(x_{m}+a\right) \operatorname{Re}\left(\frac{\partial v}{\partial x_{m}} \bar{g}\right) \mathrm{d} x-\int_{\Omega_{+}} g \bar{v} \mathrm{~d} x
\end{aligned}
$$

$H^{2}$ regularity of a solution $u \in H^{1}\left(\Omega_{+}\right)$is satisfied, e.g., if $n^{2} \in C^{0,1}\left(\overline{\Omega_{+}}\right)$and $\Gamma \in C_{\mathrm{b}}^{1,1}$.

In the following theorem, we consider right-hand sides in the space

$$
\tilde{H}^{\ell}\left(\Omega_{+}\right):=\left\{g \in H^{\ell}\left(\Omega_{+}\right), g=\left.g^{*}\right|_{\Omega_{+}} \text {for some } g^{*} \in H^{\ell}\left(\Omega_{+} \cup \overline{U_{a}^{+}}\right) \text {such that } \operatorname{supp}\left(g^{*}\right) \subset \overline{\Omega_{+}}\right\} .
$$

Theorem 3.1. Assume that $n^{2} \in C^{0,1}\left(\overline{\Omega_{+}}, \mathbb{R}\right), \partial n^{2} / \partial x_{m} \leq 0$ in $\Omega_{+}$and that $\Gamma \in C_{\mathrm{b}}^{1,1}$. For $g \in L^{2}\left(\Omega_{+}\right)$there is unique radiating variational solution $u \in H^{1}\left(\Omega_{+}\right)$of $\Delta u+k^{2} n^{2} u=g$ in $\Omega_{+}$subject to the Dirichlet boundary condition $u=h$ on $\Gamma$, that is, $u$ satisfies

$$
\int_{\Omega_{+}}\left(\nabla u \cdot \nabla \bar{v}-k^{2} n^{2} u \bar{v}\right) \mathrm{d} x-\int_{\Gamma_{a}} \bar{v} T_{n_{+}^{2}}^{+}(u) \mathrm{d} s=-\int_{\Omega_{+}} g \bar{v} \mathrm{~d} x
$$

for all $v \in H_{0}^{1}\left(\Omega_{+}\right):=\left\{v \in H^{1}\left(\Omega_{+}\right), v=0\right.$ on $\left.\Gamma\right\}$ and the boundary condition $\left.u\right|_{\Gamma}=h$ in the trace sense. 
(a) For $h=0$ there holds $\|u\|_{H^{1}\left(\Omega_{+}\right)}+\|\partial u / \partial \nu\|_{L^{2}(\Gamma)} \leq C\|g\|_{L^{2}\left(\Omega_{+}\right)}$.

(b) Let $\ell \in \mathbb{N}_{0}, n^{2} \in C^{\ell, 1}\left(\overline{\Omega_{+}}\right)$and $\Gamma \in C_{\mathrm{b}}^{\ell+1,1}$. Under the smoothness assumptions $g \in \tilde{H}^{\ell}\left(\Omega_{+}\right)$and $h \in H^{\ell+3 / 2}(\Gamma)$, elliptic regularity results imply that $\|u\|_{H^{\ell+2}\left(\Omega_{+}\right)} \leq C\left(\|g\|_{H^{\ell}\left(\Omega_{+}\right)}+\|h\|_{H^{\ell+3 / 2}(\Gamma)}\right)$.

Proof. Let us abbreviate the variational formulation (3.2) as $a(u, v)=-\int_{\Omega_{+}} g \bar{v} \mathrm{~d} x$ for all $v \in H_{0}^{1}\left(\Omega_{+}\right)$. The existence statement for $h=0$ is shown as in [11], Section 7. It is a consequence of an a priori bound $\|u\|_{H^{1}(\Omega)} \leq$ $C\|g\|_{L^{2}\left(\Omega_{+}\right)}$for any solution $u \in H_{0}^{1}\left(\Omega_{+}\right)$of (3.2) gained by the Rellich identity (3.1). See also [2] for the case $n^{2} \equiv 1$. The regularity result for $h=0$ in part (a) of the theorem is a direct consequence of the Rellich identity. The existence result for arbitrary $h$ is shown as in the standard proof for inhomogeneous elliptic boundary value problems [12], Theorem 4.10. Therefore we note that the a priori bound $\|u\|_{H^{1}(\Omega)} \leq C\|g\|_{L^{2}\left(\Omega_{+}\right)}$even implies an a priori bound for a larger class of right-hand sides [2], Lemma 4.5. Denote by $\left(H_{0}^{1}\left(\Omega_{+}\right)\right)^{*}$ the space of bounded linear functionals on $\left(H_{0}^{1}\left(\Omega_{+}\right)\right)^{*}$ with obvious norm, let $G \in\left(H_{0}^{1}\left(\Omega_{+}\right)\right)^{*}$ and assume that $u \in H_{0}^{1}\left(\Omega_{+}\right)$solves $a(u, v)=G(v)$ for all $v \in H_{0}^{1}\left(\Omega_{+}\right)$. Then $\|u\|_{H^{1}(\Omega)} \leq C\|G\|_{\left(H_{0}^{1}\left(\Omega_{+}\right)\right)^{*}}$ (with a different constant $C$ compared to the above $L^{2}$ bound).

The regularity statement in the general case follows from the corresponding regularity result for bounded domains [12], Theorem 4.18, and a technique already introduced in [11]. For sake of completeness we shall hereafter sketch some details of the proof. By abuse of notation we do not distinguish between a solution $u \in H^{1}\left(\Omega_{+}\right)$of the Helmholtz equation satisfying $\partial u / \partial \nu=T_{n_{+}^{2}}^{+}(u)$ on $\Gamma_{a}$ and its unique radiating extension to $\Omega_{+} \cup \overline{U_{a}^{+}}$.

Consider the open cube $Q=(-2,2)^{m-1} \times(0, a)$ and set $Q_{j}:=j+Q$ for $j \in(3 \mathbb{Z})^{m-1}$. The cubes $Q_{j}$ cover $\Omega$ :

$$
\Omega \subset \bigcup_{j \in(3 \mathbb{Z})^{m-1}} Q_{j}
$$

By $Q_{j}^{2}:=j+2 Q$ we denote an even larger cube containing $Q_{j}$. Set $\tilde{\Omega}_{+}=\Omega_{+} \cup \overline{U_{a}^{+}}$. From the boundary regularity result [12], Theorem 4.18, we obtain the following estimate in each cube $Q_{j}$,

$$
\|u\|_{H^{\ell+2}\left(Q_{j} \cap \Omega_{+}\right)} \leq C_{j}\left(\|u\|_{H^{1}\left(Q_{j}^{2} \cap \tilde{\Omega}_{+}\right)}+\|u\|_{H^{\ell+3 / 2}\left(Q_{j}^{2} \cap \Gamma\right)}+\|g\|_{H^{\ell}\left(Q_{j}^{2} \cap \tilde{\Omega}_{+}\right)}\right)
$$

The constants $C_{j}$ depend on $\ell$, the local smoothness of the coefficient $n^{2}$ and the local regularity of $\Gamma$. Hence, the lemma's assumptions $\Gamma \in C_{\mathrm{b}}^{\ell+1,1}$ and $n^{2} \in C^{\ell, 1}\left(\overline{\Omega_{+}}\right)$imply a uniform bound $C$ for the numbers $C_{j}$. Now we can exploit that the $Q_{j}$ cover $\Omega$,

$$
\begin{aligned}
\|u\|_{H^{\ell+2}\left(\Omega_{+}\right)} & \leq C \sum_{j \in(3 \mathbb{Z})^{m-1}}\|u\|_{H^{\ell+2}\left(Q_{j}^{2} \cup \tilde{\Omega}_{+}\right)} \\
& \leq C\left(\sum_{j \in(3 \mathbb{Z})^{m-1}}\|u\|_{H^{1}\left(Q_{j}^{2} \cap \Omega_{+}\right)}+\|u\|_{H^{\ell+3 / 2}\left(Q_{j}^{2} \cap \Gamma\right)}+\|g\|_{H^{\ell}\left(Q_{j}^{2} \cap \Omega_{+}\right)}\right) \\
& \leq C\left(\|u\|_{H^{1}\left(\left\{f(\tilde{x})<x_{m}<2 a\right\}\right)}+\|u\|_{H^{\ell+3 / 2}(\Gamma)}+\|g\|_{H^{\ell}\left(\Omega_{+}\right)}\right) .
\end{aligned}
$$

This estimate allows to prove the stated regularity result by an induction argument.

Our restriction to a real-valued refractive index $n^{2}$ is motivated by our problem setting; reference [11] shows that the last theorem holds as well for complex valued indices.

Several times in this text, for instance in the proof of Theorem 3.4, we rely on the boundedness of a weak solution operator.

Corollary 3.2 ( $L^{2}$ solution operator). Assume that $n^{2} \in C^{0,1}\left(\overline{\Omega_{+}}, \mathbb{R}\right)$ and that $\Gamma \in C_{\mathrm{b}}^{0,1}$. For a weak solution $u \in H^{1}\left(\Omega_{+}\right)$of the Helmholtz equation $\Delta u+k^{2} n^{2} u=0$ in $\Omega_{+}$which takes boundary values $h \in H^{1 / 2}(\Gamma)$ and 
satisfies the radiation condition $\partial u / \partial \nu=T_{n_{+}^{2}}^{+}(u)$ on $\Gamma_{a}$, it holds that $\|u\|_{L^{2}\left(\Omega_{+}\right)} \leq C\|h\|_{L^{2}(\Gamma)}$ for $C$ independent of $u$. Hence, the solution operator $h \mapsto u$ has a bounded extension from $L^{2}(\Gamma)$ to $L^{2}\left(\Omega_{+}\right)$.

For bounded Lipschitz domains, this is a well known result [12], Theorem 4.25. Since subsequently we only rely on a weak solution operator corresponding to $\Gamma \in C_{\mathrm{b}}^{1,1}$ (see Lem. 3.3), we merely indicate the necessary steps to transfer the proof of Corollary 3.2 from the bounded to the unbounded Lipschitz setting. One first establishes a bound $\|\partial u / \partial \nu\|_{L^{2}(\Gamma)} \leq C\|u\|_{H^{1}\left(\Omega_{+}\right)}+C\|g\|_{L^{2}\left(\Omega_{+}\right)}$for an $H^{2}$ solution of $\Delta u+k^{2} n^{2} u=g$ in $\Omega$ such that $u=0$ on $\Gamma$ and $\partial u / \partial \nu=T_{n_{+}^{2}}^{+}(u)$ on $\Gamma_{a}$ [12], Theorem 2.24. In our case this follows from the Rellich identity (3.1). We note that for general second-order elliptic operators, it is a generalized Rellich identity which is employed for this task [12], Lemma 4.22. Afterwards, careful approximation of $\Gamma$ by a smooth surface shows that this bound even holds for $H^{1}$ solutions of the problem. The proof given in [12], Theorem 2.24, carries over to our setting, with minor modifications. In the second step, one uses a duality argument (see the proof of Lem. 3.3 below) to get the bound on $u$ indicated in the corollary.

Lemma 3.3 (weak solution operator). Assume that $n^{2} \in C^{0,1}\left(\overline{\Omega_{+}}, \mathbb{R}\right)$ and that $\Gamma \in C_{\mathrm{b}}^{1,1}$. For a weak solution $u \in H^{1}\left(\Omega_{+}\right)$of the Helmholtz equation $\Delta u+k^{2} n^{2} u=0$ in $\Omega_{+}$which takes boundary values $h \in H^{1 / 2}(\Gamma)$ and satisfies the radiation condition $\partial u / \partial \nu=T_{n_{+}^{2}}^{+}(u)$ on $\Gamma_{a}$, it holds that $\|u\|_{L^{2}\left(\Omega_{+}\right)} \leq C\|h\|_{H^{-1 / 2}(\Gamma)}$ for $C$ independent of $u$. Hence, the solution operator $h \mapsto u$ has a bounded extension from $H^{-1 / 2}(\Gamma)$ to $L^{2}\left(\Omega_{+}\right)$.

Proof. Due to Theorem 3.1 there is a unique weak solution $u \in H^{1}\left(\Omega_{+}\right)$of the problem

$$
\Delta u+k^{2} n^{2} u=0 \quad \text { in } \Omega_{+}, \quad \frac{\partial u}{\partial \nu}=T_{n_{+}^{2}}^{+}(u) \quad \text { on } \Gamma_{a}, \quad u=h \quad \text { on } \Gamma,
$$

for all $h \in H^{1 / 2}(\Gamma)$, and $\|u\|_{H^{1}\left(\Omega_{+}\right)} \leq C\|h\|_{H^{1 / 2}(\Gamma)}$ for $C$ independent of $u$. Moreover, Theorem 3.1 states that for arbitrary $g \in L^{2}\left(\Omega_{+}\right)$there is a unique solution $w \in H_{0}^{1}\left(\Omega_{+}\right)$of the problem

$$
\Delta w+k^{2} n^{2} w=g \quad \text { in } \Omega_{+}, \quad \frac{\partial w}{\partial \nu}=T_{n_{+}^{2}}^{+}(u) \quad \text { on } \Gamma_{a}, \quad w=0 \quad \text { on } \Gamma,
$$

with $\|w\|_{H^{1}\left(\Omega_{+}\right)}+\|\partial w / \partial \nu\|_{L^{2}(\Gamma)} \leq C\|g\|_{L^{2}\left(\Omega_{+}\right)}$, again for $C$ independent of $w$. Our regularity assumptions are even enough to conclude that $\|w\|_{H^{2}\left(\Omega_{+}\right)} \leq C\|g\|_{L^{2}\left(\Omega_{+}\right)}$. Choose a cut-off function $\zeta \in C_{0}^{\infty}(\mathbb{R})$ such that $\zeta(t)=0$ for $t>0, \zeta(t)=1$ for $t<-1$ and $0 \leq \zeta \leq 1$. We set $\chi_{R}(x)=\zeta(|\tilde{x}|-R-1)$. Twice applying Green's first identity yields

$$
\begin{aligned}
0 & =-\int_{\Omega_{+}}\left(\Delta u+k^{2} n^{2} u\right) \chi_{R} \bar{w} \mathrm{~d} x=\int_{\Omega_{+}}\left(\nabla u \cdot \nabla\left(\bar{w} \chi_{R}\right)-k^{2} n^{2} u \chi_{R} \bar{w}\right) \mathrm{d} s-\int_{\partial \Omega_{+}} \frac{\partial u}{\partial \nu} \chi_{R} \bar{w} \mathrm{~d} s \\
& =-\int_{\Omega_{+}} u\left(\Delta\left(\chi_{R} \bar{w}\right)+k^{2} n^{2} \chi_{R} \bar{w}\right) \mathrm{d} s+\int_{\partial \Omega_{+}} u \frac{\partial\left(\chi_{R} \bar{w}\right)}{\partial \nu} \mathrm{d} s-\int_{\partial \Omega_{+}} \frac{\partial u}{\partial \nu} \chi_{R} \bar{w} \mathrm{~d} s .
\end{aligned}
$$

Since $u \in H^{1}\left(\Omega_{+}\right)$and $w \in H^{2}\left(\Omega_{+}\right)$Lebesgue's dominated convergence theorem implies

$$
\int_{\Omega_{+}} u\left(\Delta\left(\chi_{R} \bar{w}\right)-k^{2} n^{2} \chi_{R} \bar{w}\right) \mathrm{d} s \stackrel{R \rightarrow \infty}{\rightarrow} \int_{\Omega_{+}} u\left(\Delta \bar{w}-k^{2} n^{2} \bar{w}\right) \mathrm{d} s,
$$

and since $\partial \bar{w} / \partial \nu \in L^{2}(\partial \Omega)$

$$
\int_{\partial \Omega_{+}} u \frac{\partial\left(\chi_{R} \bar{w}\right)}{\partial \nu} \mathrm{d} s \stackrel{R \rightarrow \infty}{\rightarrow} \int_{\partial \Omega_{+}} u \frac{\partial \bar{w}}{\partial \nu} \mathrm{d} s
$$

as well. Obviously, $\int_{\partial \Omega_{+}} \partial u / \partial \nu \chi_{R} \bar{w} \mathrm{~d} s=\int_{\Gamma_{a}} \partial u / \partial \nu \chi_{R} \bar{w} \mathrm{~d} s$. Moreover, $\partial u / \partial \nu \in L^{2}\left(\Gamma_{a}\right)$, which can either be seen using elliptic regularity results as in the proof of Theorem 3.1, or by Fourier arguments as in [2], Lemma 2.2. 
Therefore, we also have

and we conclude that

$$
\int_{\partial \Omega_{+}} \frac{\partial u}{\partial \nu} \chi_{R} \bar{w} \mathrm{~d} s \stackrel{R \rightarrow \infty}{\rightarrow} \int_{\partial \Omega_{+}} \frac{\partial u}{\partial \nu} \bar{w} \mathrm{~d} s
$$

$$
\begin{aligned}
0 & =-\int_{\Omega_{+}} u\left(\Delta \bar{w}+k^{2} n^{2} \bar{w}\right) \mathrm{d} s+\int_{\Gamma} u \frac{\partial \bar{w}}{\partial \nu} \mathrm{d} s-\int_{\Gamma_{a}} \frac{\partial u}{\partial \nu} \bar{w} \mathrm{~d} s+\int_{\Gamma_{a}} u \frac{\partial \bar{w}}{\partial \nu} \mathrm{d} s \\
& =-\int_{\Omega_{+}} u \bar{g} \mathrm{~d} s+\int_{\Gamma} h \frac{\partial \bar{w}}{\partial \nu} \mathrm{d} s+2 \mathrm{i} \operatorname{Im} \int_{\Gamma_{a}} u \frac{\partial \bar{w}}{\partial \nu} \mathrm{d} s .
\end{aligned}
$$

We set $g=u$, take the real part of the latter equation and obtain

$$
\|u\|_{L^{2}\left(\Omega_{+}\right)}^{2} \leq\|h\|_{H^{-1 / 2}(\Gamma)}\|\partial w / \partial \nu\|_{H^{1 / 2}(\Gamma)} \leq C\|h\|_{H^{-1 / 2}(\Gamma)}\|w\|_{H^{2}\left(\Omega_{+}\right)} \leq C\|h\|_{H^{-1 / 2}(\Gamma)}\|u\|_{L^{2}\left(\Omega_{+}\right)} .
$$

Now we give an existence result for a generalized impedance boundary value problem of the form (2.14). In fact, all impedance problems we encounter during our asymptotic analysis in the following sections can be treated by the following theorem. As a general model, we consider the equation

$$
\Delta u^{\varepsilon}+k^{2} n^{2} u^{\varepsilon}=g \quad \text { in } \Omega_{+}, \quad \frac{\partial u^{\varepsilon}}{\partial \nu}=T_{n_{+}^{2}}^{+}\left(u^{\varepsilon}\right) \quad \text { on } \Gamma_{a}, \quad u^{\varepsilon}+D_{\varepsilon}\left(\frac{\partial u^{\varepsilon}}{\partial \nu}\right)=h \quad \text { on } \Gamma .
$$

If $D_{\varepsilon}$ is invertible in a suitable sense, we can state the variational formulation of this problem as follows: Find $u^{\varepsilon} \in H^{1}\left(\Omega_{+}\right)$such that

$$
\int_{\Omega_{+}}\left(\nabla u^{\varepsilon} \cdot \nabla \bar{v}-k^{2} u^{\varepsilon} \bar{v}\right) \mathrm{d} s-\int_{\Gamma_{a}} \bar{v} T_{n_{+}^{2}}^{+}\left(u^{\varepsilon}\right) \mathrm{d} s+\int_{\Gamma} \bar{v} D_{\varepsilon}^{-1}\left(u^{\varepsilon}\right) \mathrm{d} s=-\int_{\Omega_{+}} g \bar{v} \mathrm{~d} x+\int_{\Gamma} \bar{v} D_{\varepsilon}^{-1} h \mathrm{~d} s
$$

for all $v \in H^{1}\left(\Omega_{+}\right)$.

Theorem 3.4. Assume that $n^{2} \in C^{0,1}\left(\overline{\Omega_{+}}, \mathbb{R}\right)$ and that $\Gamma \in C_{\mathrm{b}}^{1,1}$. Assume further that $D_{\varepsilon}: L^{2}(\Gamma) \rightarrow L^{2}(\Gamma)$ satisfies

$$
\begin{aligned}
& \left\|D_{\varepsilon} \phi\right\|_{L^{2}(\Gamma)} \leq C \varepsilon\|\phi\|_{L^{2}(\Gamma)}, \quad \operatorname{Re}\left\langle D_{\varepsilon} \phi, \phi\right\rangle \geq C \varepsilon\|\phi\|_{L^{2}(\Gamma)}^{2}, \quad \text { and } \\
& \operatorname{Im}\left\langle D_{\varepsilon} \phi, \phi\right\rangle \geq C \varepsilon\|\phi\|_{L^{2}(\Gamma)}^{2} \quad \text { for all } \phi \in L^{2}(\Gamma) \text { and } \varepsilon \in\left(0, \varepsilon_{0}\right] \text {, }
\end{aligned}
$$

where $C$ is independent of $\varepsilon \in\left(0, \varepsilon_{0}\right]$. For $g \in L^{2}\left(\Omega_{+}\right)$and $h \in L^{2}(\Gamma)$ there is a unique solution of the variational problem (5.9) which satisfies

$$
\left\|u^{\varepsilon}\right\|_{H^{1}\left(\Omega_{+}\right)}+C_{1} \sqrt{\varepsilon}\left\|\frac{\partial u^{\varepsilon}}{\partial \nu}\right\|_{L^{2}(\Gamma)} \leq C_{2}\left(\|g\|_{L^{2}(\Omega)}+\|h\|_{L^{2}(\Gamma)}\right)
$$

for $C_{1,2}$ independent of $\varepsilon \in\left(0, \varepsilon_{0}\right]$.

Proof. We consider first the case $g=0$; the general case will again follow from this special situation. Plugging in $v=u^{\varepsilon}$ into the variational formulation (3.5), we find

$$
\int_{\Omega_{+}}\left(\left|\nabla u^{\varepsilon}\right|^{2}-k^{2} n^{2}\left|u^{\varepsilon}\right|^{2}\right) \mathrm{d} x-\int_{\Gamma_{a}} \overline{u^{\varepsilon}} T_{n_{+}^{2}}^{+}\left(u^{\varepsilon}\right) \mathrm{d} s-\int_{\Gamma} \overline{u^{\varepsilon}} \frac{\partial u^{\varepsilon}}{\partial \nu} \mathrm{d} s=0 .
$$

Taking the imaginary part of this equation, we obtain

$$
\operatorname{Im} \int_{\Gamma_{a}} \overline{u^{\varepsilon}} T_{n_{+}^{2}}^{+}\left(u^{\varepsilon}\right) \mathrm{d} s+\operatorname{Im} \int_{\Gamma} \overline{u^{\varepsilon}} \frac{\partial u^{\varepsilon}}{\partial \nu} \mathrm{d} s=0
$$


Moreover, exploiting the coercivity of $\operatorname{Im} D_{\varepsilon}$ from (3.6),

$$
\operatorname{Im} \int_{\Gamma} \frac{\partial u^{\varepsilon}}{\partial \nu} \mathrm{d} s=\operatorname{Im} \int_{\Gamma} D_{\varepsilon}\left(\frac{\partial u^{\varepsilon}}{\partial \nu}\right) \frac{\overline{\partial u^{\varepsilon}}}{\partial \nu} \mathrm{d} s+\operatorname{Im} \int_{\Gamma} \bar{h} \frac{\partial u^{\varepsilon}}{\partial \nu} \mathrm{d} s \geq c \varepsilon\left\|\frac{\partial u^{\varepsilon}}{\partial \nu}\right\|_{L^{2}(\Gamma)}^{2}+\operatorname{Im} \int_{\Gamma} \bar{h} \frac{\partial u^{\varepsilon}}{\partial \nu} \mathrm{d} s
$$

Therefore, by Lemma 3.3 and the fact that $\operatorname{Im} \int_{\Gamma_{a}} \overline{u^{\varepsilon}} T_{n_{+}^{2}}^{+}\left(u^{\varepsilon}\right) \mathrm{d} s \geq 0$,

$$
\varepsilon\left\|\frac{\partial u^{\varepsilon}}{\partial \nu}\right\|_{L^{2}(\Gamma)} \leq C\|h\|_{L^{2}(\Gamma)}
$$

Due to $(3.6), D_{\varepsilon}$ is bounded on $L^{2}(\Gamma)$ with bound $C \varepsilon$ for $\varepsilon \in\left(\varepsilon, \varepsilon_{0}\right]$. Exploiting the impedance boundary condition in (3.4), we obtain

$$
\left\|u^{\varepsilon}\right\|_{L^{2}(\Gamma)}^{2} \leq 2\left\|D_{\varepsilon}\left(\partial u^{\varepsilon} / \partial \nu\right)\right\|_{L^{2}(\Gamma)}^{2}+2\|h\|_{L^{2}(\Gamma)}^{2} \leq C \varepsilon^{2}\left\|\partial u^{\varepsilon} / \partial \nu\right\|_{L^{2}(\Gamma)}^{2}+2\|h\|_{L^{2}(\Gamma)}^{2} \leq(C \varepsilon+2)\|h\|_{L^{2}(\Gamma)}^{2} .
$$

We take the real part of (3.5) where we set $v=u^{\varepsilon}$. Note that $-\operatorname{Re} \int_{\Gamma_{a}} \overline{u^{\varepsilon}} T_{n_{+}^{2}}^{+}\left(u^{\varepsilon}\right) \mathrm{d} s \geq 0$ by the representation of $T_{n_{+}^{2}}^{+}$in (1.3). Hence, Lemma 3.3 and (3.9) yield

$$
\left\|u^{\varepsilon}\right\|_{H^{1}\left(\Omega_{+}\right)}^{2}+c \varepsilon\left\|\frac{\partial u^{\varepsilon}}{\partial \nu}\right\|_{L^{2}(\Gamma)}^{2} \leq\left(1+k^{2}\left\|n^{2}\right\|_{\infty}\right)\left\|u^{\varepsilon}\right\|_{L^{2}\left(\Omega_{+}\right)}^{2}+C\|h\|_{L^{2}(\Gamma)}^{2} \leq C\left\|u^{\varepsilon}\right\|_{L^{2}(\Gamma)}^{2}+C\|h\|_{L^{2}(\Gamma)}^{2} \leq C\|h\|_{L^{2}(\Gamma)}^{2}
$$

where all constants are independent of $\varepsilon$. Hence, we conclude that

$$
\left\|u^{\varepsilon}\right\|_{H^{1}\left(\Omega_{+}\right)}+c \sqrt{\varepsilon}\left\|\frac{\partial u^{\varepsilon}}{\partial \nu}\right\|_{L^{2}(\Gamma)} \leq C\|h\|_{L^{2}(\Gamma)}
$$

for some constant $C$ independent of $\varepsilon$.

Consider now the general case $0 \neq g \in L^{2}\left(\Omega_{+}\right)$and let $u^{\varepsilon} \in H^{1}\left(\Omega_{+}\right)$be a solution to (3.5). Let $w \in$ $H^{1}\left(\Omega_{+}\right)$be the variational solution of $\Delta w+k^{2} n^{2} w=0$ in $\Omega_{+}$subject to the homogeneous Dirichlet boundary condition $\left.w\right|_{\Gamma}=0$ and the radiation condition $\partial w / \partial \nu=T_{n_{+}^{2}}^{+}(w)$ on $\Gamma_{a}$. Recall that Theorem 3.1 states that $\|w\|_{H^{1}\left(\Omega_{+}\right)}+\|\partial w / \partial \nu\|_{L^{2}(\Gamma)} \leq C\|g\|_{L^{2}\left(\Omega_{+}\right)}$. The difference $v=u^{\varepsilon}-w$ solves $\Delta v+k^{2} n^{2} v=0$ in $\Omega_{+}$, $v+D_{\varepsilon}(\partial v / \partial \nu)=h-D_{\varepsilon}(\partial w / \partial \nu)$ and $\partial v / \partial \nu=T_{n_{+}^{2}}^{+}(v)$ on $\Gamma_{a}$, again in a variational sense. The boundedness of $D_{\varepsilon}$ on $L^{2}(\Gamma)$ from (3.6) and (3.10) show that

$$
\begin{aligned}
\|v\|_{H^{1}\left(\Omega_{+}\right)}+c \sqrt{\varepsilon}\left\|\frac{\partial v}{\partial \nu}\right\|_{L^{2}(\Gamma)} & \leq C\left(\|h\|_{L^{2}(\Gamma)}+\left\|D_{\varepsilon}(\partial w / \partial \nu)\right\|_{L^{2}(\Gamma)}\right) \\
& \leq C\left(\|h\|_{L^{2}(\Gamma)}+C\|\partial w / \partial \nu\|_{L^{2}(\Gamma)}\right) \leq C\left(\|h\|_{L^{2}(\Gamma)}+C\|g\|_{L^{2}\left(\Omega_{+}\right)}\right) .
\end{aligned}
$$

We conclude by the triangle inequality that

$$
\left\|u^{\varepsilon}\right\|_{H^{1}\left(\Omega_{+}\right)}+c \sqrt{\varepsilon}\left\|\frac{\partial u^{\varepsilon}}{\partial \nu}\right\|_{L^{2}(\Gamma)} \leq C\left(\|h\|_{L^{2}(\Gamma)}+C\|g\|_{L^{2}\left(\Omega_{+}\right)}\right),
$$

which is a $H^{1}\left(\Omega_{+}\right)$a priori bound for $u$ and finishes the proof, since this a priori bound establishes existence and uniqueness of solution by the well-known inf-sup theory for variational formulations, see, e.g., [10], Chapter 2, or [2]. 
We content ourselves here to consider right-hand sides $h \in L^{2}(\Gamma)$. Even if this is sufficient for our later purposes, the natural regularity would be $h \in H^{-1 / 2}(\Gamma)$. In fact, one can also show existence of solution for the generalized impedance boundary value problem with $h \in H^{-1 / 2}(\Gamma)$. We sketch the proof here for the case $g=0$, under the additional assumption that $D_{\varepsilon}^{-1}$ is bounded on $H^{-1 / 2}(\Gamma)$ by a constant $\mu(\varepsilon)$. Assume that $u^{\varepsilon}$ solves (3.5). Taking the imaginary part of (3.5) (with $v=u^{\varepsilon}$ ) and exploiting the coercivity properties of $D_{\varepsilon}$ we arrive at

$$
\|u\|_{L^{2}(\Gamma)}^{2} \leq \operatorname{Im} \int_{\Gamma} \bar{u} D_{\varepsilon}^{-1}(u) \mathrm{d} s \leq \operatorname{Im} \int_{\Gamma} \bar{u} D_{\varepsilon}^{-1}(h) \mathrm{d} s \leq \mu(\varepsilon)\|u\|_{H^{1 / 2}(\Gamma)}\|h\|_{H^{-1 / 2}(\Gamma)} .
$$

Taking now the real part of (3.5), we get

$$
\begin{aligned}
\|u\|_{H^{1}\left(\Omega_{+}\right)}^{2} & \leq C\|u\|_{L^{2}\left(\Omega_{+}\right)}^{2}+C \mu(\varepsilon)\|u\|_{H^{1 / 2}(\Gamma)}\|h\|_{H^{-1 / 2}(\Gamma)} \\
& \leq C\|u\|_{L^{2}(\Gamma)}^{2}+C \mu(\varepsilon)\|u\|_{H^{1}\left(\Omega_{+}\right)}\|h\|_{H^{-1 / 2}(\Gamma)} \leq C \mu(\varepsilon)\|u\|_{H^{1}\left(\Omega_{+}\right)}\|h\|_{H^{-1 / 2}(\Gamma)}
\end{aligned}
$$

with $C$ independent of $\varepsilon$. The problem with this a priori bound is that $\mu(\varepsilon)$ will, in general, fail to be bounded as $\varepsilon \rightarrow 0$. This is already the case for the generalized impedance boundary condition of order 1 in (2.13), which scales like $\varepsilon$. Since it is somewhat comfortable (but not ultimately necessary) to deal with uniform solution bounds in the following asymptotic analysis, we prefer to consider impedance data $h \in L^{2}(\Gamma)$ subsequently.

To illustrate the value of the general existence result provided by Theorem 3.4, we apply it to generalized impedance boundary value problems (2.14) of orders 1 and 2. As we already mentioned, the boundary condition for order 3 needs an additional stabilization, constructed in Section 5, which is why we do not treat this case now. Essentially, the problem with $D_{\varepsilon, 3}$, given in (2.13), is that this boundary operator violates the $L^{2}$ ellipticity assumption (3.6).

Corollary 3.5. Assume that $n^{2} \in C^{0,1}\left(\overline{\Omega_{+}}, \mathbb{R}\right)$, that $\Gamma \in C_{\mathrm{b}}^{1,1}$, and that $g \in L^{2}\left(\Omega_{+}\right)$.

(a) The solution $u^{\varepsilon, 2}$ of $(2.14)$ exists in $H^{1}\left(\Omega_{+}\right)$for all $\varepsilon>0$ and satisfies $\left\|u^{\varepsilon, 1}\right\|_{H^{1}\left(\Omega_{+}\right)} \leq C\|g\|_{L^{2}\left(\Omega_{+}\right)}$with $C$ independent of $\varepsilon \in\left(0, \varepsilon_{0}\right]$ for arbitrary $\varepsilon_{0}$.

(b) There is $\varepsilon_{0}>0$ such that the solution $u^{\varepsilon, 2}$ of $(2.14)$ exists in $H^{1}\left(\Omega_{+}\right)$for all $\varepsilon \in\left(0, \varepsilon_{0}\right]$ and satisfies the bound $\left\|u^{\varepsilon, 1}\right\|_{H^{1}\left(\Omega_{+}\right)} \leq C\|g\|_{L^{2}\left(\Omega_{+}\right)}$with $C$ independent of $\varepsilon \in\left(0, \varepsilon_{0}\right]$.

Proof. In both cases, existence and uniqueness of $u^{\varepsilon, p}$ follows from Theorem 3.4 if we verify assumption (3.6).

(a) The impedance boundary condition for $p=1$ is $u^{\varepsilon, 1}+(\varepsilon / \alpha)\left(\partial u^{\varepsilon, 1} / \partial \nu\right)=0$ on $\Gamma$. The boundary operator $D_{1, \varepsilon}=\varepsilon / \alpha=\varepsilon(1+\mathrm{i}) / \sqrt{2}$ clearly satisfies (3.6).

(b) For $p=2, u^{\varepsilon, 2}+\left(\varepsilon / \alpha-\mathrm{i} H \varepsilon^{2}\right)\left(\partial u^{\varepsilon, 2} / \partial \nu\right)=0$ on $\Gamma$. Choose $\varepsilon_{0}$ such that $|H \varepsilon|<1 /(2 \sqrt{2})$ for $\varepsilon \in\left(0, \varepsilon_{0}\right]$. Then $D_{2, \varepsilon}=\varepsilon / \sqrt{2}+\mathrm{i} \varepsilon(1 / \sqrt{2}-H \varepsilon)$ satisfies

$$
\operatorname{Re} \int_{\Gamma} \bar{\phi} D_{2, \varepsilon} \phi \mathrm{d} s=\frac{\varepsilon}{\sqrt{2}}\|\phi\|_{L^{2}(\Gamma)}^{2}, \quad \operatorname{Im} \int_{\Gamma} \bar{\phi} D_{2, \varepsilon} \phi \mathrm{d} s=\varepsilon \int_{\Gamma}\left(\frac{1}{\sqrt{2}}-H \varepsilon\right)|\phi|^{2} \mathrm{~d} s \geq \frac{\varepsilon}{2 \sqrt{2}}\|\phi\|_{L^{2}(\Gamma)}^{2}
$$

for $\phi \in L^{2}(\Gamma)$ and $0<\varepsilon \leq \varepsilon_{0}$. Hence, $D_{2, \varepsilon}$ satisfies (3.6).

\section{Asymptotic analysis and error estimates for GIBCs}

Our aim in this section is to provide the basic tools for the convergence analysis of GIBCs leading to proofs of convergence of $u^{\varepsilon, p}$ to $u^{\varepsilon}$ with optimal order $p+1$ for $p \in\{0,1,2\}$. Since the case $p=3$ requires additional manipulations, we postpone the analysis of this case to the next Section 5 . Note, however, that some of the technical lemmas contained in this present section will also be used in Section 5 to treat the case $p=3$. Our main theorem in this section is the following. 
Theorem 4.1. Let $p \in\{0,1,2\}, g \in \tilde{H}^{p}\left(\Omega_{+}\right), n^{2} \in C^{p, 1}\left(\overline{\Omega_{+}}, \mathbb{R}\right)$ and $\Gamma \in C_{\mathrm{b}}^{p+1,1}$. There are constants $\varepsilon_{0}>0$ and $C(p)>0$, independent of $\varepsilon \in\left(0, \varepsilon_{0}\right]$, such that

$$
\left\|u^{\varepsilon}-u^{\varepsilon, p}\right\|_{H^{1}\left(\Omega_{+}\right)} \leq C(p) \varepsilon^{p+1}, \quad 0<\varepsilon \leq \varepsilon_{0} .
$$

The proof of this theorem requires some preparation. In the following lemma, we show that the functions $u_{+}^{p}$ of the asymptotic expansion (2.1) are well defined in $H^{1}\left(\Omega_{+}\right)$for $p \in \mathbb{N}_{0}$ if the right hand side $g$ of the Helmholtz equation (1.1) is smooth enough.

Lemma 4.2. Set $g^{0}=g \in L^{2}\left(\Omega_{+}\right)$and $g^{\ell}=0$ for $\ell=1,2, \ldots$, and let $p \in \mathbb{N}$. For $g \in \tilde{H}^{p-1}\left(\Omega_{+}\right)$, $n^{2} \in C^{p-1,1}\left(\overline{\Omega_{+}}, \mathbb{R}\right)$ and $\Gamma \in C_{\mathrm{b}}^{p, 1}$, the functions $u_{+}^{\ell}$ are well defined in $H^{1}\left(\Omega_{+}\right)$for $\ell=0,1, \ldots, p$ through the recursion

$$
\Delta u_{+}^{\ell}+k^{2} n^{2} u_{+}^{\ell}=g^{\ell} \quad \text { in } \Omega_{+}, \quad \frac{\partial u_{+}^{\ell}}{\partial \nu}=T_{n_{+}^{2}}^{+}\left(u_{+}^{\ell}\right) \quad \text { on } \Gamma_{a}, \quad u_{+}^{\ell}=u_{-}^{\ell}(\cdot, 0) \quad \text { on } \Gamma,
$$

where $u_{-}^{\ell}(\cdot, 0)$ is determined from

$$
\left(-\frac{\partial^{2}}{\partial \eta^{2}}-\mathrm{i}\right) u_{-}^{\ell}=\sum_{\ell=1}^{8} A_{\ell} u_{-}^{\ell-l} \quad \text { in } \Gamma \times \mathbb{R}_{+}, \quad \lim _{\eta \rightarrow \infty} u_{-}^{\ell}\left(x_{\Gamma}, \eta\right)=0 \quad \text { on } \Gamma,
$$

together with the coupling condition (2.5), that is,

$$
\frac{\partial u_{+}^{\ell-1}}{\partial \nu}\left(x_{\Gamma}\right)=\frac{\partial u_{-}^{\ell}}{\partial \eta}\left(x_{\Gamma}, 0\right), \quad \text { for } x_{\Gamma} \in \Gamma .
$$

Proof. For $\ell=0$ we know from $(2.6)$ that $u_{-}^{0}(\cdot, 0)=0$. Hence, $u_{+}^{0}$ solves a homogeneous Dirichlet problem for the Helmholtz equation with right hand side $g^{0}=g$. From Theorem 3.1 we conclude that $u_{+}^{0} \in H^{2}\left(\Omega_{+}\right)$ for $g \in L^{2}\left(\Omega_{+}\right)$; thus the normal derivative $\partial u_{+}^{0} / \partial \nu$ belongs to $H^{1 / 2}(\Gamma)$ and the bounds $\left\|\partial u_{+}^{0} / \partial \nu\right\|_{H^{1 / 2}\left(\Omega_{+}\right)} \leq$ $C\left\|u_{+}^{0}\right\|_{H^{2}\left(\Omega_{+}\right)} \leq C\|g\|_{L^{2}\left(\Omega_{+}\right)}$hold.

More generally, for $g \in \tilde{H}^{p-1}\left(\Omega_{+}\right)$and $n^{2} \in C^{p-1,1}\left(\overline{\Omega_{+}}, \mathbb{R}\right)$ and $\Gamma \in C_{\mathrm{b}}^{p, 1}$ as well as $\Gamma$ smooth enough we have that $u_{+}^{0} \in H^{p+1}\left(\Omega_{+}\right)$; thus the normal derivative $\partial u_{+}^{0} / \partial \nu$ belongs to $H^{p-1 / 2}(\Gamma)$ and the bounds

$$
\left\|\partial u_{+}^{0} / \partial \nu\right\|_{H^{p-1 / 2}(\Gamma)} \leq C\left\|u_{+}^{0}\right\|_{H^{p+1}\left(\Omega_{+}\right)} \leq C(p)\|g\|_{H^{p-1}\left(\Omega_{+}\right)}
$$

hold for $p=1,2, \ldots$

In consequence, the Dirichlet trace $u_{-}^{1}(\cdot, 0)$ given in $(2.7)$ is well defined in $H^{p-1 / 2}(\Gamma)$ for $g$ in $H^{p}\left(\Omega_{+}\right)$and we can solve the Dirichlet problem (4.2) in $H^{p}\left(\Omega_{+}\right)$according to Theorem 3.1, with $\left\|\partial u_{+}^{1} / \partial \nu\right\|_{H^{p-3 / 2}(\Gamma)} \leq$ $C\left\|\partial u_{+}^{1} / \partial \nu\right\|_{H^{p}(\Gamma)} \leq C\|g\|_{H^{p-1}\left(\Omega_{+}\right)}$, as long as $p-1>0$. Existence of $u_{+}^{\ell}$ for $\ell=2, \ldots, p$ follows from an induction argument. Existence and boundedness of $u_{+}^{p}$ in $H^{1}\left(\Omega_{+}\right)$and $\partial u_{+}^{p} / \partial \nu \in H^{1 / 2}(\Gamma)$ requires $g \in$ $\tilde{H}^{p-1}\left(\Omega_{+}\right)$, as well as $n^{2} \in C^{p-1,1}\left(\overline{\Omega_{+}}\right)$and $\Gamma$ of class $C_{\mathrm{b}}^{p, 1}$.

The proof of Theorem 4.1 is broken into two main steps, since we are not able to show (4.1) directly, but rather introduce $\tilde{u}^{\varepsilon, 3}$ (see (2.10)) as an intermediate term and prove that

$$
\left\|u^{\varepsilon}-u^{\varepsilon, p}\right\|_{H^{1}\left(\Omega_{+}\right)} \leq\left\|u^{\varepsilon}-\tilde{u}^{\varepsilon, p}\right\|_{H^{1}\left(\Omega_{+}\right)}+\left\|\tilde{u}^{\varepsilon, p}-u^{\varepsilon, p}\right\|_{H^{1}\left(\Omega_{+}\right)} \leq C \varepsilon^{p+1} .
$$

To treat the two differences which appear in the last equation, let us first introduce a sequel of technical lemmas. 
Lemma 4.3. Let $\Gamma \in C_{\mathrm{b}}^{0,1}$. Then there is a constant $C>0$ such that

$$
\|u\|_{L^{2}(\Gamma)}^{2} \leq C\left(\|\nabla u\|_{L^{2}\left(\Omega_{ \pm}\right)}\|u\|_{L^{2}\left(\Omega_{ \pm}\right)}+\|u\|_{L^{2}\left(\Omega_{ \pm}\right)}^{2}\right) \quad \text { for all } u \in H^{1}\left(\Omega_{ \pm}\right) .
$$

Proof. Estimates of this kind are well known from earlier works on generalized impedance boundary conditions, however, since $\Omega_{ \pm}$is unbounded we briefly sketch the proof for the domain $\Omega_{+}$.

For $u \in C^{\infty}\left(\overline{\Omega_{+}}\right) \cap H^{1}\left(\Omega_{+}\right)$, the fundamental theorem of calculus implies

$$
|u(\tilde{x}, h)|^{2}-|u(\tilde{x}, f(\tilde{x}))|^{2}=2 \int_{f(\tilde{x})}^{h} u\left(\tilde{x}, x_{m}\right) \frac{\partial u(\tilde{x}, s)}{\partial x_{m}} \mathrm{~d} s .
$$

If we define the segment $S_{\tilde{x}}:=\{y=(\tilde{x}, s), f(\tilde{x})<s<h\}$, then

$$
|u(\tilde{x}, f(\tilde{x}))|^{2} \leq 2\|u(\tilde{x}, \cdot)\|_{L^{2}\left(S_{\tilde{x}}\right)}\left\|\frac{\partial u(\tilde{x}, \cdot)}{\partial x_{m}}\right\|_{L^{2}\left(S_{\tilde{x}}\right)}+|u(\tilde{x}, h)|^{2} .
$$

Integration with respect to $h$ yields

$$
|h-f(\tilde{x})||u(\tilde{x}, f(\tilde{x}))|^{2} \leq 2|h-f(\tilde{x})|\|u(\tilde{x}, \cdot)\|_{L^{2}\left(S_{\tilde{x}}\right)}\left\|\frac{\partial u(\tilde{x}, \cdot)}{\partial x_{m}}\right\|_{L^{2}\left(S_{\tilde{x}}\right)}+\|u(\tilde{x}, h)\|_{L^{2}\left(S_{\tilde{x}}\right)}^{2}
$$

We integrate with respect to $\tilde{x}$,

$$
\int_{\mathbb{R}^{m-1}}|u(\tilde{x}, f(\tilde{x}))|^{2} \mathrm{~d} \tilde{x} \leq 2\|u\|_{L^{2}\left(\Omega_{+}\right)}\left\|\frac{\partial u}{\partial x_{m}}\right\|_{L^{2}\left(\Omega_{+}\right)}+\sup _{\tilde{x} \in \mathbb{R}^{m-1}}|h-f(\tilde{x})|^{-1}\|u\|_{L^{2}\left(\Omega_{+}\right)}^{2},
$$

which gives the claim of the lemma, since $\|u\|_{L^{2}(\Gamma)}^{2} \leq C \int_{\mathbb{R}^{m-1}}|u(\tilde{x}, f(\tilde{x}))|^{2} \mathrm{~d} \tilde{x}$ and since $C^{\infty}\left(\overline{\Omega_{+}}\right) \cap H^{1}\left(\Omega_{+}\right)$is dense in $H^{1}\left(\Omega_{+}\right)$.

Lemma 4.4. Assume that $n^{2} \in C^{0,1}\left(\overline{\Omega_{+}}, \mathbb{R}\right), \Gamma \in C_{\mathrm{b}}^{0,1}$, and that $v^{\varepsilon} \in H^{1}(\Omega)$ satisfies

$$
\Delta v^{\varepsilon}+k^{2} n^{2} v^{\varepsilon}=0 \quad \text { in } \Omega, \quad \frac{\partial v^{\varepsilon}}{\partial \nu}=T_{n_{+}^{2}}^{+}\left(v^{\varepsilon}\right) \quad \text { on } \Gamma_{a} \quad \text { for } \varepsilon \in\left(0, \varepsilon_{0}\right]
$$

together with the a priori estimate

$$
\left|\int_{\Omega}\left(\left|\nabla v^{\varepsilon}\right|^{2}-k^{2} n^{2}\left|v^{\varepsilon}\right|^{2}\right) \mathrm{d} x-\int_{\Gamma_{a}} \overline{v^{\varepsilon}} T_{n_{+}^{2}}^{+}\left(v^{\varepsilon}\right) \mathrm{d} s\right| \leq C\left(\varepsilon^{s+1 / 2}\left\|v^{\varepsilon}\right\|_{L^{2}(\Gamma)}+\varepsilon^{s}\left\|v^{\varepsilon}\right\|_{L^{2}\left(\Omega_{-}\right)}\right)
$$

for $C, s>0$ independent of $\varepsilon$ and $v^{\varepsilon}$. Then

$$
\left\|v^{\varepsilon}\right\|_{H^{1}(\Omega)} \leq C \varepsilon^{s+1}, \quad\left\|v^{\varepsilon}\right\|_{L^{2}\left(\Omega_{-}\right)} \leq C \varepsilon^{s+2}, \quad \text { and } \quad\left\|v^{\varepsilon}\right\|_{L^{2}(\Gamma)} \leq C \varepsilon^{s+3 / 2}
$$

for $\varepsilon \in\left(0, \varepsilon_{0}\right]$.

Proof. Suppose that $\lambda_{\varepsilon}=\left\|v^{\varepsilon}\right\|_{L^{2}(\Omega)} / \varepsilon^{s+1}$ is unbounded as $\varepsilon \rightarrow 0$. We set $w^{\varepsilon}=v^{\varepsilon} /\left\|v^{\varepsilon}\right\|_{L^{2}(\Omega)}$ and note that (4.4) implies

$$
\left|\int_{\Omega}\left(\left|\nabla w^{\varepsilon}\right|^{2}-k^{2} n^{2}\left|w^{\varepsilon}\right|^{2}\right) \mathrm{d} x-\int_{\Gamma_{a}} \overline{w^{\varepsilon}} T_{n_{+}^{2}}^{+}\left(w^{\varepsilon}\right) \mathrm{d} s\right| \leq \frac{C}{\lambda_{\varepsilon}}\left(\varepsilon^{-1 / 2}\left\|w^{\varepsilon}\right\|_{L^{2}(\Gamma)}+\varepsilon^{-1}\left\|w^{\varepsilon}\right\|_{L^{2}\left(\Omega_{-}\right)}\right) .
$$


As $C / \lambda_{\varepsilon}$ is bounded as $\varepsilon \rightarrow 0$, the last inequality implies an estimate for the absolute value of the imaginary part of the left hand side. Moreover, as $-k^{2} \operatorname{Im}\left(n^{2}\right) \leq 0$ and $-\operatorname{Im} \int_{\Gamma_{a}} \overline{w^{\varepsilon}} T_{n_{+}^{2}}^{+}\left(w^{\varepsilon}\right) \mathrm{d} s \leq 0$ as well, we obtain

$$
k^{2} \int_{\Omega_{-}}\left|w^{\varepsilon}\right|^{2} \mathrm{~d} x \leq \frac{C}{\lambda_{\varepsilon}}\left(\varepsilon^{3 / 2}\left\|w^{\varepsilon}\right\|_{L^{2}(\Gamma)}+\varepsilon\left\|w^{\varepsilon}\right\|_{L^{2}\left(\Omega_{-}\right)}\right) .
$$

By Lemma 4.3,

$$
\left\|w^{\varepsilon}\right\|_{L^{2}\left(\Omega_{-}\right)}^{3 / 2} \leq C \varepsilon^{3 / 2}\left(\left\|\nabla w^{\varepsilon}\right\|_{L^{2}\left(\Omega_{-}\right)}^{1 / 2}+\left\|w^{\varepsilon}\right\|_{L^{2}\left(\Omega_{-}\right)}^{1 / 2}\right)\left\|w^{\varepsilon}\right\|_{L^{2}\left(\Omega_{-}\right)}^{1 / 2}+C \varepsilon\left\|w^{\varepsilon}\right\|_{L^{2}\left(\Omega_{-}\right)}
$$

and

$$
\left\|w^{\varepsilon}\right\|_{L^{2}\left(\Omega_{-}\right)} \leq C \varepsilon^{3 / 2}\left\|\nabla w^{\varepsilon}\right\|_{L^{2}\left(\Omega_{-}\right)}^{1 / 2}+C \varepsilon\left\|w^{\varepsilon}\right\|_{L^{2}\left(\Omega_{-}\right)}^{1 / 2} \leq C \varepsilon^{3 / 2}\left\|\nabla w^{\varepsilon}\right\|_{L^{2}\left(\Omega_{-}\right)}^{1 / 2}+C \varepsilon^{3 / 2}+\frac{1}{2}\left\|w^{\varepsilon}\right\|_{L^{2}\left(\Omega_{-}\right)}^{3 / 2},
$$

where we used Young's inequality in the last step. We conclude that

$$
\left\|w^{\varepsilon}\right\|_{L^{2}\left(\Omega_{-}\right)}^{3 / 2} \leq C \varepsilon^{3 / 2}\left(1+\left\|\nabla w^{\varepsilon}\right\|_{L^{2}\left(\Omega_{-}\right)}^{1 / 2}\right) .
$$

Since $-\operatorname{Re} \int_{\Gamma_{a}} \overline{w^{\varepsilon}} T_{n_{+}^{2}}^{+}\left(w^{\varepsilon}\right) \mathrm{d} s>0$, the corresponding estimate for the real part of (4.5) yields

$$
\begin{aligned}
\int_{\Omega}\left|\nabla w^{\varepsilon}\right|^{2} \mathrm{~d} x & \leq k^{2} \int_{\Omega} \operatorname{Re}\left(n^{2}\right)\left|w^{\varepsilon}\right|^{2} \mathrm{~d} x+\frac{C}{\lambda_{\varepsilon}}\left(\varepsilon^{-1 / 2}\left\|w^{\varepsilon}\right\|_{L^{2}(\Gamma)}+\varepsilon^{-1}\left\|w^{\varepsilon}\right\|_{L^{2}\left(\Omega_{-}\right)}\right) \\
& \leq C+C\left(\varepsilon^{-1 / 2}\left\|w^{\varepsilon}\right\|_{L^{2}(\Gamma)}+\varepsilon^{-1}\left\|w^{\varepsilon}\right\|_{L^{2}\left(\Omega_{-}\right)}\right) .
\end{aligned}
$$

The term $\varepsilon^{-1 / 2}\left\|w^{\varepsilon}\right\|_{L^{2}(\Gamma)}$ can be estimated by Lemma 4.3 ,

$$
\varepsilon^{-1 / 2}\left\|w^{\varepsilon}\right\|_{L^{2}(\Gamma)} \leq 2 \varepsilon^{-1 / 2}\left\|w^{\varepsilon}\right\|_{L^{2}\left(\Omega_{-}\right)}+2 \varepsilon^{-1 / 2}\left\|w^{\varepsilon}\right\|_{L^{2}\left(\Omega_{-}\right)}^{1 / 2}\left\|\nabla w^{\varepsilon}\right\|_{L^{2}\left(\Omega_{-}\right)}^{1 / 2},
$$

which we plug into (4.7) to obtain

$$
\int_{\Omega}\left|\nabla w^{\varepsilon}\right|^{2} \mathrm{~d} x \leq C+C \varepsilon^{-1}\left\|w^{\varepsilon}\right\|_{L^{2}\left(\Omega_{-}\right)}\left(1+\left\|\nabla u^{\varepsilon}\right\|_{L^{2}\left(\Omega_{-}\right)}^{1 / 2}\right) \quad \text { for } \varepsilon \in\left(\varepsilon, \varepsilon_{0}\right] .
$$

By means of (4.6),

$$
\varepsilon^{-1}\left\|w^{\varepsilon}\right\|_{L^{2}\left(\Omega_{-}\right)} \leq C\left(1+\left\|\nabla u^{\varepsilon}\right\|_{L^{2}\left(\Omega_{-}\right)}^{1 / 3}\right)
$$

Hence, using the latter bound in (4.8) we obtain $\left\|\nabla w^{\varepsilon}\right\|_{L^{2}(\Omega)}^{2} \leq C\left(1+\left\|\nabla w^{\varepsilon}\right\|_{L^{2}\left(\Omega_{-}\right)}^{2 / 3}\right)$, which shows that $\left\|\nabla w^{\varepsilon}\right\|_{L^{2}(\Omega)}^{2}$ is uniformly bounded in $\varepsilon \in\left(0, \varepsilon_{0}\right]$. From (4.6) we conclude that $\varepsilon^{-1}\left\|w^{\varepsilon}\right\|_{L^{2}\left(\Omega_{-}\right)}$is bounded as well. Lemma 4.3 implies that $\varepsilon^{-1}\left\|w^{\varepsilon}\right\|_{L^{2}(\Gamma)}^{2}$ is bounded for $\varepsilon \in\left(0, \varepsilon_{0}\right]$. Then, however, we conclude from Lemma 3.3 that $\varepsilon^{-1}\left\|w^{\varepsilon}\right\|_{L^{2}\left(\Omega_{+}\right)}^{2}$ is bounded for $\varepsilon \in\left(0, \varepsilon_{0}\right]$. Using (4.9) we see that $\varepsilon^{-1}\left\|w^{\varepsilon}\right\|_{L^{2}\left(\Omega_{-}\right)}^{2}$ is bounded as well. However, the conclusion that $\varepsilon^{-1}\left\|w^{\varepsilon}\right\|_{L^{2}(\Omega)}^{2}$ is bounded for $\varepsilon \in\left(0, \varepsilon_{0}\right]$ is a contradiction since by construction $\left\|w^{\varepsilon}\right\|_{L^{2}(\Omega)}^{2}=1$. Hence, the bound $\left\|v^{\varepsilon}\right\|_{L^{2}(\Omega)} \leq C \varepsilon^{s+1}$ holds for $\varepsilon \in\left(0, \varepsilon_{0}\right]$. The three estimates stated in the lemma now follow from the above inequalities precisely as in the proof of [9], Lemma 5.3.

Now we proceed with an estimate for the first difference $\left\|u^{\varepsilon}-\tilde{u}^{\varepsilon, p}\right\|_{H^{1}\left(\Omega_{+}\right)}$appearing in (4.3). 
Theorem 4.5. Let $p \in \mathbb{N}_{0}$ and assume that $g \in \tilde{H}^{p}\left(\Omega_{+}\right), n^{2} \in C^{p, 1}\left(\overline{\Omega_{+}}, \mathbb{R}\right)$ and $\Gamma \in C_{\mathrm{b}}^{p+1,1}$. Then there is $\varepsilon_{0}>0$ and a constant $C(p)$ independent of $\varepsilon$ such that

$$
\left\|u^{\varepsilon}-\tilde{u}^{\varepsilon, p}\right\|_{H^{1}\left(\Omega_{+}\right)} \leq C(p) \varepsilon^{p+1} \quad \text { for } \varepsilon \in\left(0, \varepsilon_{0}\right] .
$$

Proof. We recall the definition of $\tilde{u}^{\varepsilon, p}$ in (2.10), the cut off function $\chi$ and the coordinates $x=\left(x_{\Gamma}, \nu\right)$ in $\Omega_{-}$, both introduced in Section 2, and define

$$
\tilde{u}_{\chi}^{\varepsilon, p}(x)= \begin{cases}\sum_{\ell=0}^{p} \varepsilon^{\ell} u_{+}^{\ell}(x), & x \in \Omega_{+}, \quad p \in \mathbb{N}_{0} . \\ \chi(x) \sum_{\ell=0}^{p} \varepsilon^{\ell} u_{-}^{\ell}\left(x_{\Gamma}, q / \varepsilon\right), & x \in \Omega_{-},\end{cases}
$$

We will first prove an estimate for the difference $\left\|u^{\varepsilon}-\tilde{u}_{\chi}^{\varepsilon, p}\right\|_{H^{1}(\Omega)}$, where $\tilde{u}_{\chi}^{\varepsilon, p}$ has been defined in (4.10). Since $\tilde{u}_{\chi}^{\varepsilon, p}=\tilde{u}^{\varepsilon, p}$ in $\Omega_{+}$, the estimate stated in the theorem will follow.

In the proof of [9], Lemma 5.1, the authors show that the error $\mathrm{e}^{\varepsilon, p}:=u^{\varepsilon}-\tilde{u}_{\chi}^{\varepsilon, p}$ satisfies the following transmission problem,

$\Delta e^{\varepsilon, p}+k^{2} n^{2} e^{\varepsilon, p}=0 \quad$ in $\Omega_{+}, \quad \frac{\partial e^{\varepsilon, p}}{\partial \nu}=T_{n_{+}^{2}}^{+}\left(e^{\varepsilon, p}\right) \quad$ on $\Gamma_{a}$

$$
[u]_{\Gamma}=0, \quad\left[\frac{\partial u}{\partial \nu}\right]_{\Gamma}=\left.\varepsilon^{p} \frac{\partial u_{+}^{p}}{\partial \nu}\right|_{\Gamma}, \quad \Delta e^{\varepsilon, p}+k^{2} n^{2} e^{\varepsilon, p}=g^{\varepsilon, p} \quad \text { in } \Omega_{-},
$$

where

$$
g^{\varepsilon, p}(x)=-\varepsilon^{p-1} \chi \sum_{\ell=1}^{8} \sum_{j=0}^{\ell-1} \varepsilon^{p} A_{\ell-j-1} u_{-}^{k+j+1-\ell}\left(x_{\Gamma}, q / \varepsilon\right)+2 \nabla \chi \cdot \sum_{\ell=0}^{p} \varepsilon^{\ell} \nabla u_{-}^{\ell}+\Delta \chi \sum_{\ell=0}^{p} \varepsilon^{\ell} \nabla u_{-}^{\ell},
$$

for $x=x_{\Gamma}+q \nu \in \Omega_{-}$. The computations leading to the form of $g^{\varepsilon, p}$ are literally the same as in [9]. Also, the proof that $\left\|g^{\varepsilon, p}\right\|_{L^{2}\left(\Omega_{-}\right)} \leq C \varepsilon^{p-1 / 2}$ can be achieved as in that reference. The variational formulation for $e^{\varepsilon, p}$ in (4.11), obtained with the help of Green's first identity, is

$$
\int_{\Omega}\left(\nabla e^{\varepsilon, p} \cdot \nabla \bar{v}-k^{2} n^{2} e^{\varepsilon, p} \bar{v}\right) \mathrm{d} x-\int_{\Gamma_{a}} \bar{v} T_{n_{+}^{2}}^{+}\left(e^{\varepsilon, p}\right) \mathrm{d} s=\varepsilon^{p} \int_{\Gamma} \frac{\partial u_{+}^{p}}{\partial \nu} \bar{v} \mathrm{~d} s-\int_{\Omega_{-}} g^{\varepsilon, p} \bar{v} \mathrm{~d} s
$$

for all $v \in H^{1}(\Omega)$. Plugging in $v=e^{\varepsilon, p}$ we find that

$$
\begin{aligned}
\left|\int_{\Omega}\left(\left|\nabla e^{\varepsilon, p}\right|^{2}-k^{2} n^{2}\left|e^{\varepsilon, p}\right|^{2}\right) \mathrm{d} x-\int_{\Gamma_{a}} \bar{v} T_{n_{+}^{2}}^{+}\left(e^{\varepsilon, p}\right) \mathrm{d} s\right| \leq \varepsilon^{p}\left\|\frac{\partial u_{+}^{p}}{\partial \nu}\right\|_{L^{2}(\Gamma)}\left\|e^{\varepsilon, p}\right\|_{L^{2}(\Gamma)} \\
+\left\|g^{\varepsilon, p}\right\|_{L^{2}\left(\Omega_{-}\right)}\left\|e^{\varepsilon, p}\right\|_{L^{2}\left(\Omega_{-}\right)} \leq C \varepsilon^{p}\left\|e^{\varepsilon, p}\right\|_{L^{2}(\Gamma)}+C \varepsilon^{p-1 / 2}\left\|e^{\varepsilon, p}\right\|_{L^{2}\left(\Omega_{-}\right)}
\end{aligned}
$$

due to Lemma 4.2. From this estimate, we conclude by Lemma 4.4 that $\left\|e^{\varepsilon, p}\right\|_{H^{1}(\Omega)} \leq C \varepsilon^{p+1 / 2},\left\|e^{\varepsilon, p}\right\|_{L^{2}\left(\Omega_{-}\right)} \leq$ $C \varepsilon^{p+3 / 2}$ and $\left\|e^{\varepsilon, p}\right\|_{L^{2}(\Gamma)} \leq C \varepsilon^{p+1}$ for $p \in \mathbb{N}_{0}$. Therefore we can finally estimate

$$
\begin{aligned}
\left\|u^{\varepsilon}-\tilde{u}^{\varepsilon, p}\right\|_{H^{1}\left(\Omega_{+}\right)} & =\left\|u^{\varepsilon}-\tilde{u}^{\varepsilon, p+1}+\varepsilon^{p+1} u_{+}^{p+1}\right\|_{H^{1}\left(\Omega_{+}\right)}=\left\|u^{\varepsilon}-\tilde{u}_{\chi}^{\varepsilon, p+1}+\varepsilon^{p+1} u_{+}^{p+1}\right\|_{H^{1}\left(\Omega_{+}\right)} \\
& \leq\left\|u^{\varepsilon}-\tilde{u}_{\chi}^{\varepsilon, p+1}\right\|_{H^{1}\left(\Omega_{+}\right)}+\varepsilon^{p+1}\left\|u_{+}^{p+1}\right\|_{H^{1}\left(\Omega_{+}\right)} \leq C \varepsilon^{p+3 / 2}+C \varepsilon^{p+1} .
\end{aligned}
$$

For the latter bound we exploited Lemma 4.2 stating that $u^{p+1}$ is well defined in $H^{1}\left(\Omega_{+}\right)$for data $g \in \tilde{H}^{p}\left(\Omega_{+}\right)$, $n^{2} \in C^{p, 1}\left(\overline{\Omega_{+}}\right)$and $\Gamma \in C_{\mathrm{b}}^{p+1,1}$. 
Now we finish this section with the proof of the main theorem, Theorem 4.1, showing the convergence $u^{\varepsilon, p} \rightarrow u^{\varepsilon}$ in $H^{1}\left(\Omega_{+}\right)$of optimal order $p+1$ for $p=0,1,2$.

Proof of Theorem 4.1. In view of (4.3) we only need to show that $\left\|\tilde{u}^{\varepsilon, p}-u^{\varepsilon, p}\right\|_{H^{1}\left(\Omega_{+}\right)} \leq C \varepsilon^{p+1}$. By (2.11) and Lemma 4.2, the difference $e^{\varepsilon, p}=\tilde{u}^{\varepsilon, p}-u^{\varepsilon, p} \in H^{1}\left(\Omega_{+}\right)$solves

$\Delta e^{\varepsilon, p}+k^{2} n^{2} e^{\varepsilon, p}=0 \quad$ in $\Omega_{+}, \quad \frac{\partial e^{\varepsilon, p}}{\partial \nu}=T_{n_{+}^{2}}^{+}\left(e^{\varepsilon, p}\right) \quad$ on $\Gamma_{a}, \quad$ and $\quad e^{\varepsilon, p}+D_{\varepsilon, p}\left(\frac{\partial e^{\varepsilon, p}}{\partial \nu}\right)=\varepsilon^{p+1} r^{\varepsilon, p} \quad$ on $\Gamma$

where $r^{\varepsilon, p}$ is given by (2.12). By Lemma 4.2 we observe that $\left\|r^{\varepsilon, p}\right\|_{H^{1 / 2}(\Gamma)} \leq C(p)$ for $p=0,1,2$. Now, Theorem 3.5 yields the claim of Theorem 4.1 .

\section{Stabilized GIBC OF ORDER 3}

Let us now start to investigate the Neumann-to-Dirichlet impedance boundary condition of order 3 , with the aim to prove an analogous convergence result as in Theorem 4.1 for $p=3$. As we saw in Section 2 , formal expansion yields the following candidate for a Neumann-to-Dirichlet impedance condition of order 3 ,

$$
u^{\varepsilon}+\frac{\varepsilon \sqrt{2}}{2}\left((1+\mathrm{i})-\sqrt{2} \mathrm{i} \varepsilon H-(1-\mathrm{i}) \frac{\varepsilon^{2}}{2}\left(3 H^{2}-G+k^{2}+\Delta_{\Gamma}\right)\right) \frac{\partial u^{\varepsilon}}{\partial \nu}=0 \quad \text { on } \Gamma .
$$

The difficulty with this boundary condition is that the operator applied to the normal derivative is a tangential differential operator of order 2. In contrast, the exact Neumann-to-Dirichlet operator is a pseudodifferential operator of order -1 , mapping $H^{-1 / 2}(\Gamma)$ continuously into $H^{1 / 2}(\Gamma)$. Also, the boundary condition in (5.1) violates the $L^{2}$ coercivity assumption (3.6), since $\operatorname{Im} \int_{\Gamma} \overline{u^{\varepsilon}}\left(\partial u^{\varepsilon} / \partial \nu\right) \mathrm{d} s$ is indefinite. Our subsequent modifications of (5.1), inspired by the analysis of a corresponding problem in a bounded setting [9], yield a condition of order 3 that satisfies (3.6). Note that these modifications are not crucial for the analysis of the bounded setting [9]. In contrast, they seem to be crucial in our unbounded context, since the compactness arguments for the analysis of (5.1) for a bounded obstacle from [9] certainly do not apply for our problem.

First considering all real terms of the boundary operator in (5.1), we formally compute that

$$
\frac{\varepsilon \sqrt{2}}{2}\left(1-\frac{\varepsilon^{2}}{2}\left(3 H^{2}-G+k^{2}+\Delta_{\Gamma}\right)\right)=\frac{\varepsilon \sqrt{2}}{2}\left(1-\frac{\varepsilon^{2}}{2}\left(3 H^{2}-G+k^{2}\right)-\frac{\varepsilon^{2}}{2}\left(1-\varepsilon^{2} \Delta_{\Gamma}\right)^{-1} \Delta_{\Gamma}\right)+\mathcal{O}\left(\varepsilon^{4}\right)
$$

since $\Delta_{\Gamma}-\left(I-\varepsilon^{2} \Delta_{\Gamma}\right)^{-1} \Delta_{\Gamma}=-\varepsilon^{2} \Delta_{\Gamma}^{2}\left(I-\varepsilon^{2} \Delta_{\Gamma}\right)^{-1}$. We note that $1-\varepsilon^{2} \Delta_{\Gamma}$ is bounded and coercive on $H^{1}(\Gamma)$, hence invertible due to Lax-Milgram's theorem, and that the term on the right constitutes a tangential differential operator on $\Gamma$ of order 0 . Next, we use a Padé approximation to change the sign of the surface Laplacian appearing in the complex terms of (5.1). Using that $\left(1-\varepsilon^{2} \Delta_{\Gamma}\right)\left(1+\varepsilon^{2} \Delta_{\Gamma}\right)=1-\varepsilon^{4} \Delta_{\Gamma}^{2}$, we find

$$
\begin{aligned}
\frac{\varepsilon \sqrt{2}}{2}\left(1-\varepsilon \sqrt{2} H+\frac{\varepsilon^{2}}{2}\left(3 H^{2}-G+k^{2}+\right.\right. & \left.\left.\Delta_{\Gamma}\right)\right)=\frac{\varepsilon \sqrt{2}}{2}\left(\frac{1}{2}-\varepsilon \sqrt{2} H+\frac{\varepsilon^{2}}{2}\left(3 H^{2}-G+k^{2}\right)+\frac{1}{2}+\frac{\varepsilon^{2}}{2} \Delta_{\Gamma}\right) \\
= & \frac{\varepsilon \sqrt{2}}{2}\left(\frac{1}{2}-\varepsilon \sqrt{2} H+\frac{\varepsilon^{2}}{2}\left(3 H^{2}-G+k^{2}\right)+\frac{1}{2}\left(1-\varepsilon^{2} \Delta_{\Gamma}\right)^{-1}\right)+\mathcal{O}\left(\varepsilon^{4}\right)
\end{aligned}
$$


Therefore we define a modified boundary operator $\hat{D}_{\varepsilon, 3}$ for a generalized impedance boundary condition of order 3 as follows,

$$
\begin{aligned}
\hat{D}_{\varepsilon, 3} \phi= & \frac{\varepsilon \sqrt{2}}{2}\left(1-\frac{\varepsilon^{2}}{2}\left(3 H^{2}-G+k^{2}\right)-\frac{\varepsilon^{2}}{2}\left(1-\varepsilon^{2} \Delta_{\Gamma}\right)^{-1} \Delta_{\Gamma}\right) \phi \\
& +\frac{\mathrm{i} \varepsilon \sqrt{2}}{2}\left(\frac{1}{2}-\varepsilon \sqrt{2} H+\frac{\varepsilon^{2}}{2}\left(3 H^{2}-G+k^{2}\right)+\frac{1}{2}\left(I-\varepsilon^{2} \Delta_{\Gamma}\right)^{-1}\right) \phi .
\end{aligned}
$$

Using this operator, we define $u^{\varepsilon, 3}$ via the following boundary value problem

$$
\Delta u^{\varepsilon, 3}+k^{2} n^{2} u^{\varepsilon, 3}=g \quad \text { in } \Omega_{+}, \quad \frac{\partial u^{\varepsilon, 3}}{\partial \nu}=T_{n_{+}^{2}}^{+}\left(u^{\varepsilon, 3}\right) \quad \text { on } \Gamma_{a}, u^{\varepsilon, 3}+\hat{D}_{\varepsilon, 3}\left(\partial u^{\varepsilon, 3} / \partial \nu\right)=0 \quad \text { on } \Gamma \text {. }
$$

Three important properties of $\hat{D}_{\varepsilon, 3}$ are collected in the next lemma, namely boundedness and coercivity on $L^{2}(\Gamma)$, which results of in invertibility of $\hat{D}_{\varepsilon, 3}$ on $L^{2}(\Gamma)$.

Lemma 5.1. There are constants $\varepsilon_{0}>0$ and $C$ independent of $\varepsilon>0$ such that

$$
\begin{aligned}
& \left\|\hat{D}_{\varepsilon, 3} \phi\right\|_{L^{2}(\Gamma)} \leq C \varepsilon\|\phi\|_{L^{2}(\Gamma)}, \quad \operatorname{Re}\left\langle\hat{D}_{\varepsilon, 3} \phi, \phi\right\rangle \geq C \varepsilon\|\phi\|_{L^{2}(\Gamma)}^{2}, \quad \text { and } \\
& \operatorname{Im}\left\langle\hat{D}_{\varepsilon, 3} \phi, \phi\right\rangle \geq C \varepsilon\|\phi\|_{L^{2}(\Gamma)}^{2} \quad \text { for all } \phi \in L^{2}(\Gamma) \text { and } \varepsilon \in\left(0, \varepsilon_{0}\right] .
\end{aligned}
$$

Proof. We start with some preparations. First, the identity $\left(I-\varepsilon^{2} \Delta_{\Gamma}\right)\left(1-\varepsilon^{2} \Delta_{\Gamma}\right)^{-1} \phi=\phi$ for $\phi \in L^{2}(\Gamma)$ implies by one partial integration that

$$
\int_{\Gamma}\left(\varepsilon^{2} \nabla_{\Gamma}\left(\left(1-\varepsilon^{2} \Delta_{\Gamma}\right)^{-1} \phi\right) \cdot \nabla_{\Gamma} \bar{\psi}+\left(\left(1-\varepsilon^{2} \Delta_{\Gamma}\right)^{-1} \phi\right) \bar{\psi}\right) \mathrm{d} s=\int_{\Gamma}\left(1-\varepsilon^{2} \Delta_{\Gamma}\right)^{-1} \phi \bar{\psi} \mathrm{d} s
$$

for all $\psi \in H^{1}(\Gamma)$. Setting $\psi=\left(1-\varepsilon^{2} \Delta_{\Gamma}\right)^{-1} \phi$ and taking the complex conjugate of (5.4) we obtain

$$
\left\langle\left(I-\varepsilon^{2} \Delta_{\Gamma}\right)^{-1} \phi, \phi\right\rangle=\left\|\left(I-\varepsilon^{2} \Delta_{\Gamma}\right)^{-1} \phi\right\|_{L^{2}(\Gamma)}^{2}+\varepsilon^{2}\left\|\nabla_{\Gamma}\left(I-\varepsilon^{2} \Delta_{\Gamma}\right)^{-1} \phi\right\|_{L^{2}(\Gamma)}^{2}
$$

as well as setting $\psi=\Delta_{\Gamma}\left(1-\varepsilon^{2} \Delta_{\Gamma}\right)^{-1}$ results by further integrations by parts in

$$
-\left\langle\Delta_{\Gamma}\left(I-\varepsilon^{2} \Delta_{\Gamma}\right)^{-1} \phi, \phi\right\rangle=\left\|\nabla_{\Gamma}\left(I-\varepsilon^{2} \Delta_{\Gamma}\right)^{-1} \phi\right\|_{L^{2}(\Gamma)}^{2}+\varepsilon^{2}\left\|\Delta_{\Gamma}\left(I-\varepsilon^{2} \Delta_{\Gamma}\right)^{-1} \phi\right\|_{L^{2}(\Gamma)}^{2} .
$$

The Cauchy-Schwarz inequality applied to (5.4) shows that $\left\|\left(I-\varepsilon^{2} \Delta_{\Gamma}\right)^{-1} \phi\right\|_{L^{2}(\Gamma)}^{2} \leq\|\phi\|_{L^{2}(\Gamma)}^{2}$. Therefore (5.5) implies

$$
\varepsilon^{2}\left\|\nabla_{\Gamma}\left(I-\varepsilon^{2} \Delta_{\Gamma}\right)^{-1} \phi\right\|_{L^{2}(\Gamma)}^{2} \leq\|\phi\|_{L^{2}(\Gamma)}^{2} .
$$

The identity $-\varepsilon^{2} \Delta_{\Gamma}\left(I-\varepsilon^{2} \Delta_{\Gamma}\right)^{-1}=I-\left(I-\varepsilon^{2} \Delta_{\Gamma}\right)^{-1}$ and (5.5) finally shows that $-\varepsilon^{2}\left\langle\Delta_{\Gamma}\left(I-\varepsilon^{2} \Delta_{\Gamma}\right)^{-1} \phi, \phi\right\rangle \leq$ $\|\phi\|_{L^{2}(\Gamma)}^{2}$ as well, and hence (5.6) yields $\varepsilon^{4}\left\|\Delta_{\Gamma}\left(I-\varepsilon^{2} \Delta_{\Gamma}\right)^{-1} \phi\right\|_{L^{2}(\Gamma)}^{2} \leq\|\phi\|_{L^{2}(\Gamma)}^{2}$.

A brief look at the definition of $\hat{D}_{\varepsilon, 3}$ shows that we can write

$$
\hat{D}_{\varepsilon, 3}=\varepsilon\left(a_{\varepsilon}-\sqrt{2} \varepsilon^{2} / 4\left(1-\varepsilon^{2} \Delta_{\Gamma}\right)^{-1} \Delta_{\Gamma}\right)+\mathrm{i} \varepsilon\left(b_{\varepsilon}+\frac{1}{2}\left(I-\varepsilon^{2} \Delta_{\Gamma}\right)^{-1}\right)
$$

and that we observe that there is $\varepsilon_{0}>0$ such that the coefficients $a_{\varepsilon}$ and $b_{\varepsilon}$ on $\Gamma$ are bounded between two constants $0<c_{0}<C$ uniformly in $\varepsilon \in\left(0, \varepsilon_{0}\right]$. Consequently,

$$
\left\langle\hat{D}_{\varepsilon, 3} \phi, \psi\right\rangle=\varepsilon\left\langle a_{\varepsilon} \phi, \psi\right\rangle-\frac{\sqrt{2} \varepsilon^{3}}{4}\left\langle\left(1-\varepsilon^{2} \Delta_{\Gamma}\right)^{-1} \Delta_{\Gamma} \phi, \psi\right\rangle+\mathrm{i} \varepsilon\left\langle b_{\varepsilon} \phi, \psi\right\rangle-\frac{\mathrm{i} \varepsilon}{2}\left\langle\left(I-\varepsilon^{2} \Delta_{\Gamma}\right)^{-1} \phi, \psi\right\rangle
$$


for all $\psi \in L^{2}(\Gamma)$ and for $\psi=\phi$ we can estimate real and imaginary part of this expression from below due to our above computations, $c_{0}\|\phi\|_{L^{2}(\Gamma)}^{2} \leq \operatorname{Re}\left\langle\hat{D}_{\varepsilon, 3} \phi, \phi\right\rangle$ and $c_{0}\|\phi\|_{L^{2}(\Gamma)}^{2} \leq \operatorname{Im}\left\langle\hat{D}_{\varepsilon, 3} \phi, \phi\right\rangle$. For an upper bound on $L^{2}(\Gamma)$, we set $\psi=\hat{D}_{\varepsilon, 3} \phi$, which yields by the Cauchy-Schwarz inequality

$$
\left\|\hat{D}_{\varepsilon, 3} \phi\right\|_{L^{2}(\Gamma)} \leq C \varepsilon\|\phi\|_{L^{2}(\Gamma)}+C \varepsilon^{3}\left\|\left(I-\varepsilon^{2} \Delta_{\Gamma}\right)^{-1} \Delta_{\Gamma} \phi\right\|_{L^{2}(\Gamma)}+C \varepsilon\left\|\left(I-\varepsilon^{2} \Delta_{\Gamma}\right)^{-1} \phi\right\|_{L^{2}(\Gamma)} \leq C \varepsilon\|\phi\|_{L^{2}(\Gamma)} .
$$

Remark 5.2. If $\Gamma \in C_{\mathrm{b}}^{\ell, 1}$ for any $\ell \in \mathbb{N}$, then $\hat{D}_{\varepsilon, 3}$ is bounded on $H^{s}(\Gamma)$ for arbitrary $s \in \mathbb{R}$. Indeed, then the curvatures $H$ and $G$ are smooth functions on $\Gamma$ and $\Delta_{\Gamma}^{s}\left(a_{\varepsilon} \phi\right)=\tilde{a}_{\varepsilon} \Delta_{\Gamma}^{s} \phi$ for a smooth function $\tilde{a}_{\varepsilon}$ that is bounded by some constant independent of $\varepsilon$. Analogously, $\Delta_{\Gamma}^{s}\left(b_{\varepsilon} \phi\right)=\tilde{b}_{\varepsilon} \Delta_{\Gamma}^{s} \phi$. Further, $\Delta_{\Gamma}^{s}\left(1-\varepsilon^{2} \Delta_{\Gamma}\right)^{-1}=\left(1-\varepsilon^{2} \Delta_{\Gamma}\right)^{-1} \Delta_{\Gamma}^{s}$ and therefore

$$
\begin{aligned}
\left\langle\Delta_{\Gamma}^{s} \hat{D}_{\varepsilon, 3} \phi, \Delta_{\Gamma}^{s} \psi\right\rangle= & \varepsilon\left\langle\tilde{a}_{\varepsilon} \Delta_{\Gamma}^{s} \phi, \Delta_{\Gamma}^{s} \psi\right\rangle-\frac{\sqrt{2} \varepsilon^{3}}{4}\left\langle\left(1-\varepsilon^{2} \Delta_{\Gamma}\right)^{-1} \Delta_{\Gamma}^{s+1} \phi, \Delta_{\Gamma}^{s} \psi\right\rangle \\
& +\mathrm{i} \varepsilon\left\langle\tilde{b}_{\varepsilon} \Delta_{\Gamma}^{s} \phi, \Delta_{\Gamma}^{s} \psi\right\rangle-\frac{\mathrm{i} \varepsilon}{2}\left\langle\left(I-\varepsilon^{2} \Delta_{\Gamma}\right)^{-1} \Delta_{\Gamma}^{s} \phi, \Delta_{\Gamma}^{s} \psi\right\rangle
\end{aligned}
$$

Taking $\psi=\hat{D}_{\varepsilon, 3} \phi$ we find $\left\|\Delta_{\Gamma}^{s} \hat{D}_{\varepsilon, 3} \phi\right\|_{L^{2}(\Gamma)} \leq C \varepsilon\left\|\Delta_{\Gamma}^{s} \phi\right\|_{L^{2}(\Gamma)}$ for $\phi \in H^{2 s}(\Gamma)$ with $C$ independent of $\varepsilon$. We conclude by interpolation in the scale of Sobolev spaces between $L^{2}(\Gamma)$ and $H^{2 s}(\Gamma)$ and a duality argument.

In combination with the abstract existence result from Theorem 3.4, the last lemma easily shows existence of a solution $u^{\varepsilon, 3} \in H^{1}\left(\Omega_{+}\right)$to the generalized impedance boundary value problem

$$
\Delta u+k^{2} u=g \quad \text { in } \Omega_{+}, \quad \frac{\partial u}{\partial \nu}=T_{n_{+}^{2}}^{+}(u) \quad \text { on } \Gamma_{a}, \quad u+\hat{D}_{\varepsilon, 3}\left(\frac{\partial u}{\partial \nu}\right)=h \quad \text { on } \Gamma .
$$

Before stating this existence result in the following corollary, we note that the variational form of the problem reads

$$
\int_{\Omega_{+}}\left(\nabla u \cdot \nabla \bar{v}-k^{2} u \bar{v}\right) \mathrm{d} s-\int_{\Gamma_{a}} \bar{v} T_{n_{+}^{2}}^{+}(u) \mathrm{d} s+\int_{\Gamma} \bar{v} \hat{D}_{\varepsilon, 3}^{-1}(u) \mathrm{d} s=-\int_{\Omega_{+}} g \bar{v} \mathrm{~d} x+\int_{\Gamma} \bar{v} \hat{D}_{\varepsilon, 3}^{-1} h \mathrm{~d} s
$$

for all $v \in H^{1}\left(\Omega_{+}\right)$. Note that $\hat{D}_{\varepsilon, 3}^{-1}$ is indeed boundedly invertible on $L^{2}(\Gamma)$ for $\varepsilon$ in some interval $\left(0, \varepsilon_{0}\right]$ due to its coercivity.

Corollary 5.3. For $g \in L^{2}\left(\Omega_{+}\right)$and $h \in L^{2}(\Gamma)$ there is a unique solution $u^{\varepsilon, 3} \in H^{1}\left(\Omega_{+}\right)$of the variational problem (5.9) which satisfies

$$
\left\|u^{\varepsilon, 3}\right\|_{H^{1}\left(\Omega_{+}\right)}+C_{1} \sqrt{\varepsilon}\left\|\frac{\partial u^{\varepsilon, 3}}{\partial \nu}\right\|_{L^{2}(\Gamma)} \leq C_{2}\left(\|g\|_{L^{2}\left(\Omega_{+}\right)}+\|h\|_{L^{2}(\Gamma)}\right)
$$

for $C_{1,2}$ independent of $\varepsilon \in\left(0, \varepsilon_{0}\right]$.

Now we prove our main result of this section, which proves order optimal error estimate for the approximation error $\left\|u^{\varepsilon}-u^{\varepsilon, 3}\right\|_{H^{1}\left(\Omega_{+}\right)}$. Of course, we use several of the technical lemmas contained in the previous Section 4. The error estimate given here requires severe smoothness assumptions on the data $g, n^{2}$ and $\Gamma$ and we state those assumptions explicitly.

Theorem 5.4. Assume that $g \in \tilde{H}^{7}\left(\Omega_{+}\right), n^{2} \in C^{7,1}\left(\overline{\Omega_{+}}, \mathbb{R}\right)$ and that $\Gamma \in C_{\mathrm{b}}^{8,1}$. Then there are constants $\varepsilon_{0}>0$ and $C>0$ independent of $\varepsilon \in\left(0, \varepsilon_{0}\right]$ such that

$$
\left\|u^{\varepsilon}-u^{\varepsilon, 3}\right\|_{H^{1}\left(\Omega_{+}\right)} \leq C \varepsilon^{4} .
$$


Proof. As in (4.3) we split the quantity we need to estimate in two parts,

$$
\left\|u^{\varepsilon}-u^{\varepsilon, 3}\right\|_{H^{1}\left(\Omega_{+}\right)} \leq\left\|u^{\varepsilon}-\tilde{u}^{\varepsilon, 3}\right\|_{H^{1}\left(\Omega_{+}\right)}+\left\|\tilde{u}^{\varepsilon, 3}-u^{\varepsilon, 3}\right\|_{H^{1}\left(\Omega_{+}\right)} .
$$

The first part has been already treated in Lemma 4.5. For the second part, we note that $e^{\varepsilon, 3}:=\tilde{u}^{\varepsilon, 3}-u^{\varepsilon, 3}$ solves

$$
\Delta e^{\varepsilon, 3}+k^{2} n^{2} e^{\varepsilon, 3}=0 \quad \text { in } \Omega_{+}, \quad \frac{\partial e^{\varepsilon, 3}}{\partial \nu}=T_{n_{+}^{2}}^{+}\left(e^{\varepsilon, 3}\right) \quad \text { on } \Gamma_{a}, \quad \text { and } \quad e^{\varepsilon, 3}+\hat{D}_{\varepsilon, 3}\left(\frac{\partial e^{\varepsilon, k}}{\partial \nu}\right)=\varepsilon^{4} \hat{r}^{\varepsilon, 3} \quad \text { on } \Gamma .
$$

The right hand side $\hat{r}^{\varepsilon, 3}$ is different from $r^{\varepsilon, 3}$ given in (2.12), since $\hat{r}^{\varepsilon, 3}$ relies on the modified boundary operator $\hat{D}_{\varepsilon, 3}$ instead of $D_{\varepsilon, 3}$. More precisely,

$$
\varepsilon^{4} \hat{r}^{\varepsilon, 3}=\varepsilon^{4} r^{\varepsilon, 3}+\left(\hat{D}_{\varepsilon, 3}-D_{\varepsilon, 3}\right)\left(\frac{\partial \tilde{u}^{\varepsilon, 3}}{\partial \nu}\right)
$$

From the definition (2.12) and Lemma 4.2 it follows that $\left\|r^{\varepsilon, 3}\right\|_{L^{2}(\Gamma)}$ is bounded by some constant independent of $\varepsilon$. However, the difference $\hat{D}_{\varepsilon, 3}-D_{\varepsilon, 3}$ is, modulo constants independent of $\varepsilon$, sum of the two terms

$$
\varepsilon^{2} \Delta_{\Gamma}-\varepsilon^{2}\left(I-\varepsilon^{2} \Delta_{\Gamma}\right)^{-1} \Delta_{\Gamma}=-\varepsilon^{4} \Delta_{\Gamma}^{2}\left(I-\varepsilon^{2} \Delta_{\Gamma}\right)^{-1}
$$

and

$$
1+\varepsilon^{2} \Delta_{\Gamma}-\left(1-\varepsilon^{2} \Delta_{\Gamma}\right)^{-1}=\varepsilon^{4} \Delta_{\Gamma}^{2}\left(1-\varepsilon^{2} \Delta_{\Gamma}\right)^{-1} .
$$

Thus, to show that $\left\|\left(\hat{D}_{\varepsilon, 3}-D_{\varepsilon, 3}\right)\left(\partial \tilde{u}^{\varepsilon, 3} / \partial \nu\right)\right\|_{L^{2}(\Gamma)} \leq C$ for $C$ independent of $\varepsilon$, we need to prove that

$$
\left\|\Delta_{\Gamma}^{2}\left(1-\varepsilon^{2} \Delta_{\Gamma}\right)^{-1}\left(\partial \tilde{u}^{\varepsilon, 3} / \partial \nu\right)\right\|_{L^{2}(\Gamma)}=\left\|\left(1-\varepsilon^{2} \Delta_{\Gamma}\right)^{-1} \Delta_{\Gamma}^{2}\left(\partial \tilde{u}^{\varepsilon, 3} / \partial \nu\right)\right\|_{L^{2}(\Gamma)}
$$

is bounded independent of $\varepsilon$. In the proof of Lemma 5.1 we showed that $\left\|\left(I-\varepsilon \Delta_{\Gamma}\right)^{-1} \phi\right\|_{L^{2}(\Gamma)} \leq\|\phi\|_{L^{2}(\Gamma)}$. Therefore it is sufficient to show that $\left\|\Delta_{\Gamma}^{2}\left(\partial \tilde{u}^{\varepsilon, 3} / \partial \nu\right)\right\|_{L^{2}(\Gamma)}$ is bounded independent of $\varepsilon$, that is, $\left\|\partial \tilde{u}^{\varepsilon, 3} / \partial \nu\right\|_{H^{4}(\Gamma)}<C$. Lemma 4.2 shows that such a bound is guaranteed if $g, n^{2}$ and $\Gamma$ are smooth enough; for our purpose, $g \in \tilde{H}^{7}\left(\Omega_{+}\right), n^{2} \in C_{\mathrm{b}}^{7,1}\left(\Omega_{+}\right)$and $\Gamma \in C_{\mathrm{b}}^{8,1}$ are sufficient. If all data is smooth enough, an application of Theorem 3.4 finishes the proof.

\section{NumERICAL EXPERIMENTS}

This section is dedicated to the presentation of some numerical results that underline the usefulness of the derived GIBCs, especially the interest in the condition of order 3. Our experiments are restricted to the 2-D case. Since we treated rough surface scattering problems by variational methods, we opted for the use of finite element methods to compute the numerical solution using the FreeFem++ library (http://www.freefem.org/ $f f++)$. The major difficulty linked to the use of volumic methods is the truncation of the computational domain. One possible approach is the use of Dirichlet-to-Neumann maps introduced in the second section of this paper. The second possibility, adopted here, is the use of so-called perfectly matched absorbing layers (PML) (see Fig. 2). We refer to [3] for a description of this method in the context of rough surface scattering problems. However, the available approximation theory so far only considers horizontal truncation of the computational domain - little is known on stability and approximation of a fully discrete problem posed on a bounded domain. Therefore, the numerical results presented in the following intend to only give first numerical validations and also hints on numerical difficulties that need to be addressed in future works.

Following [3], we used a PML which covers the three exterior sides of the computational domain. Again, there is no theory validating this choice; the PML method can only be justified if an infinite horizontal layer is used above the rough surface. However, the option we used gave better results than, e.g., simply imposing 


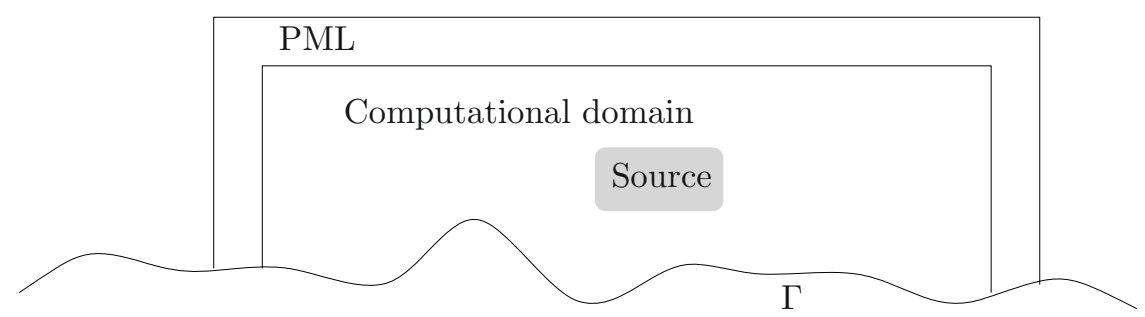

FIGURE 2. The numerical experiments settings.
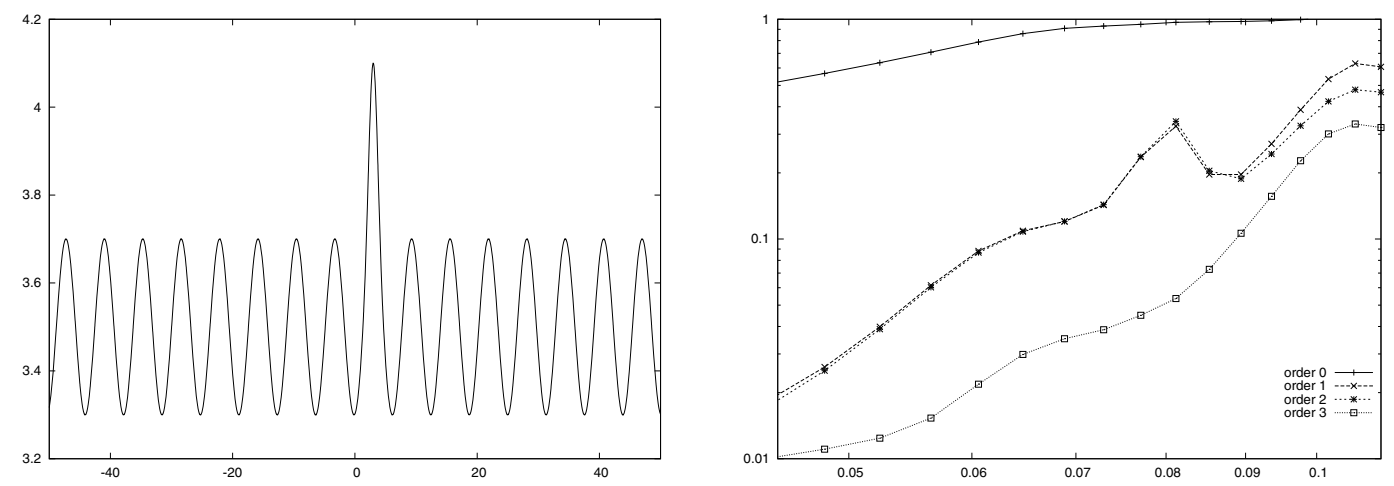

Figure 3. Left: surface profile: $f(t)=3.5+0.2 \cos (t-3)+0.4 \exp \left(-(t-3)^{2}\right)$. Right: relative $L^{2}$ error versus $\varepsilon$ (log-log scale) for GIBCs of order $0,1,2$ and 3.

Dirichlet boundary conditions at the vertical cut-off. The choice of the width of the computational domain in the horizontal direction is certainly a key point for the accuracy of the approximate solutions. This is evident from the slow decay (proportional to $\left|x_{1}\right|^{-3 / 2}$ ) of the fundamental solution of the Helmholtz equation in a half space with Dirichlet boundary conditions.

The two examples shown in Figures 3 and 4 correspond to a computational domain which is $100 \lambda$ large in horizontal direction, where $\lambda=2 \pi / k$ (=1 in our cases). When approximating the absorbing medium scattering problem (1.4) we work on a domain that is $5 \lambda$ thick. The PML width is $\lambda$ and we use an (optimized) linear profile for the absorption coefficient going from 1 at the inner boundary of the PML to $\sigma_{0}=5.75+3 i$ at the outer boundary (see [3]). The numerical examples are computed using $P 2$ finite elements. A reference solution is computed using a fine mesh of the two layered medium.

We observe in both experiments that the use of higher order GIBCs significantly improves the accuracy of the approximate model. For all GIBCs, the error decreases with respect to $\varepsilon$. However, we failed in obtaining confirmation of convergence rates predicted by our theory. This seems to be mainly due to the numerical error induced by lateral bounds of the computational domain. Finer meshes for the computation of the reference solutions did not change the results considerably. We observed that the residual error due to this truncation cannot be made smaller than $1 \%$, which would also explain why the three curves (for GIBCs) meet for small $\varepsilon$. We therefore think that the design of an efficient method to bound the computational domain constitutes one important step before going deeper in the numerical validation of these approximate boundary conditions. Addressing this issue is far beyond the scope of the present work. 

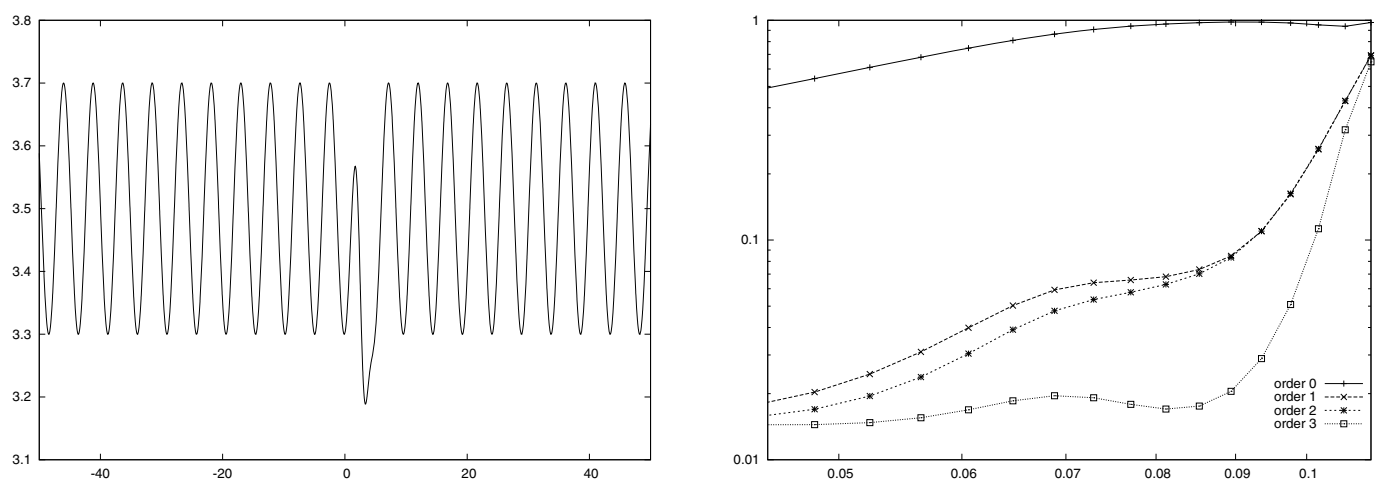

Figure 4. Left: surface profile: $f(t)=3.5+0.2 \cos (1.3 t-3)-0.4 \exp \left(-(t-3)^{2}\right)$. Right: relative $L^{2}$ error versus $\varepsilon$ (log-log scale) for GIBCs of order $0,1,2$ and 3 .

We end this discussion by noticing that when comparing the two examples, one observes that better results are obtained for rough surfaces with less sharp variations, which is somehow a predictable behavior since more regularity is needed for higher order GIBCs. Also, the difference between the conditions of order 1 and 2 is not too large, which is in accordance with theory, since the surface oscillation in our examples is small compared to the wavelength.

\section{REFERENCES}

[1] M. Artola and M. Cessenat, Diffraction d'une onde électromagnétique par un obstacle borné à permittivité et perméabilité élevées. C. R. Acad. Sci. Paris Sér. I Math. 314 (1992) 349-354.

[2] S.N. Chandler-Wilde and P. Monk, Existence, uniqueness, and variational methods for scattering by unbounded rough surfaces. SIAM. J. Math. Anal. 37 (2005) 598-618.

[3] S.N. Chandler-Wilde and P. Monk, The pml for rough surface scattering. Appl. Numer. Math. 59 (2009) 2131-2154.

[4] S.N. Chandler-Wilde and C.R. Ross, Scattering by rough surfaces: the Dirichlet problem for the Helmholtz equation in a non-locally perturbed half-plane. Math. Meth. Appl. Sci. 19 (1996) 959-976.

[5] S.N. Chandler-Wilde, E. Heinemeyer and R. Potthast, Acoustic scattering by mildly rough surfaces in three dimensions. SIAM J. Appl. Math. 66 (2006) 1002-1026.

[6] S.N. Chandler-Wilde, P. Monk and M. Thomas, The mathematics of scattering by unbounded, rough, inhomogeneous layers. J. Comput. Appl. Math. 204 (2007) 549-559.

[7] P. Cummings and X. Feng, Sharp regularity coefficient estimates for complex-valued acoustic and elastic Helmholtz equations. Math. Mod. Meth. Appl. Sci. 16 (2006) 139-160.

[8] M. Duruflé, H. Haddar and P. Joly, Higher order generalized impedance boundary conditions in electromagnetic scattering problems. C. R. Phys. 7 (2006) 533-542.

[9] H. Haddar, P. Joly and H.-M. Nguyen, Generalized impedance boundary conditions for scattering by strongly absorbing obstacles: the scalar case. Math. Mod. Meth. Appl. Sci. 15 (2005) 1273-1300.

[10] F. Ihlenburg, Finite element analysis of acoustic scattering. Springer (1998).

[11] A. Lechleiter and S. Ritterbusch, A variational method for wave scattering from penetrable rough layers. IMA J. Appl. Math. (2009) doi:10.1093/imamat/hxp040.

[12] W. McLean, Strongly Elliptic Systems and Boundary Integral Operators. Cambridge University Press, Cambridge (2000).

[13] J.C. Nédélec, Acoustic and electromagnetic equations, Applied Mathematical Sciences 144. Springer-Verlag, Berlin (2001).

[14] S.M. Rytov, Calcul du skin-effet par la méthode des perturbations. J. Phys. USSR 2 (1940) 233-242.

[15] T.B.A. Senior and J.L. Volakis, Approximate boundary conditions in electromagnetics, IEE Electromagnetic waves series 41. The institution of Electrical Engineers, London (1995).

[16] B. Zhang and S.N. Chandler-Wilde, Integral equation methods for scattering by infinite rough surfaces. Math. Meth. Appl. Sci. 26 (2003) 463-488. 